IZA DP No. 8996

Innovation, Inequality and a Golden Rule for Growth in an Economy with Cobb-Douglas Function and an R\&D Sector

Paul J.J. Welfens

April 2015 


\title{
Innovation, Inequality and a Golden Rule for Growth in an Economy with Cobb-Douglas Function and an R\&D Sector
}

\author{
Paul J.J. Welfens \\ EIIW and Schumpeter School, University of Wuppertal, \\ Sciences Po, IZA and AICGS/Johns Hopkins University
}

Discussion Paper No. 8996

April 2015

IZA

P.O. Box 7240

53072 Bonn

Germany

Phone: +49-228-3894-0

Fax: +49-228-3894-180

E-mail: iza@iza.org

Any opinions expressed here are those of the author(s) and not those of IZA. Research published in this series may include views on policy, but the institute itself takes no institutional policy positions. The IZA research network is committed to the IZA Guiding Principles of Research Integrity.

The Institute for the Study of Labor (IZA) in Bonn is a local and virtual international research center and a place of communication between science, politics and business. IZA is an independent nonprofit organization supported by Deutsche Post Foundation. The center is associated with the University of Bonn and offers a stimulating research environment through its international network, workshops and conferences, data service, project support, research visits and doctoral program. IZA engages in (i) original and internationally competitive research in all fields of labor economics, (ii) development of policy concepts, and (iii) dissemination of research results and concepts to the interested public.

IZA Discussion Papers often represent preliminary work and are circulated to encourage discussion. Citation of such a paper should account for its provisional character. A revised version may be available directly from the author. 


\section{ABSTRACT \\ Innovation, Inequality and a Golden Rule for Growth in an Economy with Cobb-Douglas Function and an R\&D Sector}

The innovative approach presented introduces a modified neoclassical growth model which includes a new bias of technological progress in a quasi-endogenous growth model in which part of labor is used in the research \& development sector. The combination of a macroeconomic production function and a new progress function, plus the assumption that the output elasticity of capital is positively influenced by the size of the R\&D sector, sheds new light on innovation and growth as well as income inequality: Thus there is a new approach for explaining Piketty's historical findings of a medium term rise of the capital income share in industrialized countries - both in the earlier and later part of the 19th century and in 1990-2010. A rising share of capital income can be explained within this approach by the increase in the output elasticity of capital, which has been developed in a new way, namely in the context of R\&D. In the approach presented herein, the golden rule issues are also highlighted and it is shown that choosing the right size of the R\&D sector will bring about maximum sustainable per capita consumption. While the basic new model is presented for the case of a closed economy, one could easily accommodate both trade and foreign direct investment and thereby get a better understanding of complex international investment, trade and FDI dynamics - including with respect to the envisaged Transatlantic Trade and Investment Partnership.

JEL Classification: O11, O32, O40, D63

Keywords: innovation, growth, inequality, golden rule, Piketty

Corresponding author:

Paul J.J. Welfens

University of Wuppertal

Rainer-Gruenter-Str. 21

42119 Wuppertal

Germany

E-mail:welfens@eiiw.uni-wuppertal.de

\footnotetext{
* I am grateful for the excellent research support of Tony Irawan, Vladimir Udalov, Samir Kadiric, David Hanrahan and Jens Perret, EIIW; I also appreciate discussion with Robert Lawrence, Kennedy School/Harvard University, particularly on the links between trade and innovation dynamics and the perspectives for TTIP. The usual disclaimer applies.
} 


\section{Table of Contents}

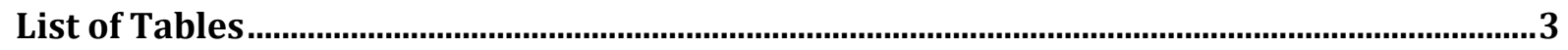

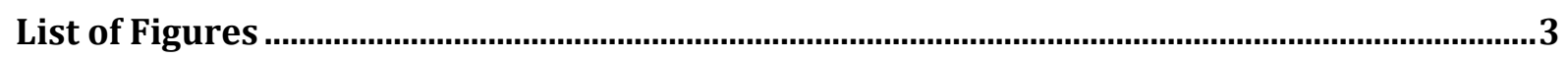

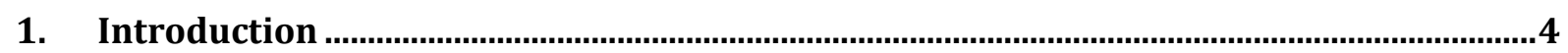

2. New Quasi-Endogenous Growth Model With Biased Technological Progress .............. 15

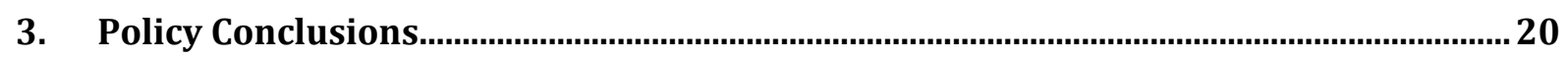

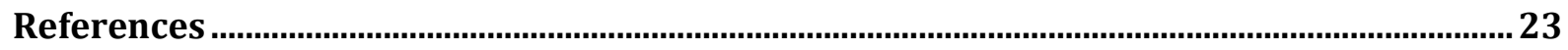

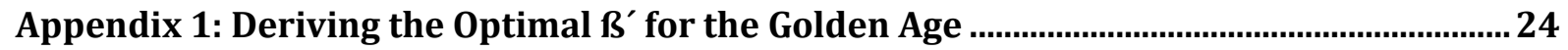

Appendix 2: Perspectives on Meeting the Golden Rule in Selected Countries (difference

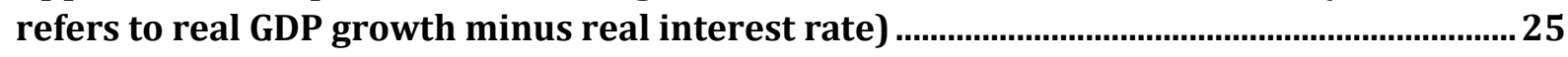

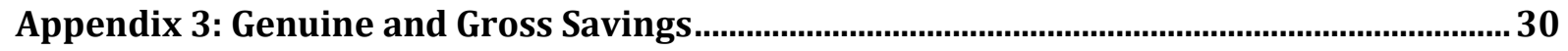

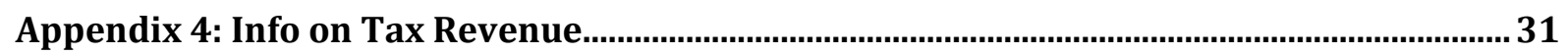

\section{List of Tables}

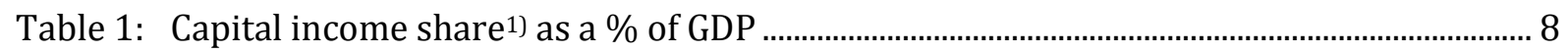

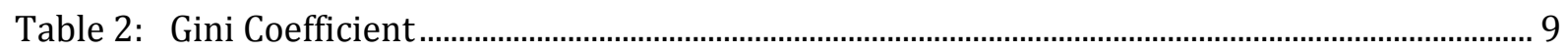

Table 3: Imported R\&D services from abroad which is used as intermediate input (as a \% of

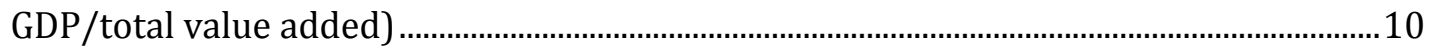

Table 4: Total R\&D services which is used as intermediate input (as a $\%$ of GDP/total value added).

Table 5: Total Domestic R\&D services which is used as intermediate input (as a \% of GDP/total value added) ............................................................................................................................ 12

Table 6: Total intramural R\&D expenditure (GERD) [as a \% of GDP] …….......................................13

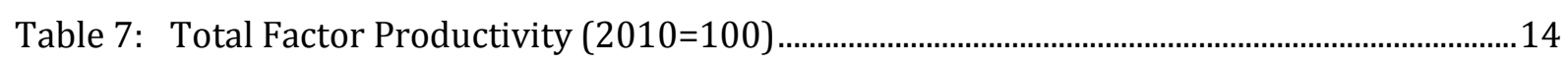

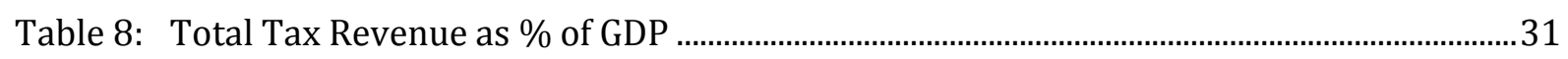

\section{List of Figures}

Figure 1: Rise of the R\&D Sector in the Quasi-Neoclassical Growth Model.. 


\section{Introduction}

The role of economic growth and income distribution is a key field of Economics and since Schumpeter has been linked to innovation dynamics. In a macroeconomic perspective there are key challenges with respect to taxation and innovation policy as well as with respect to the general framework conditions for economic agents; the size of innovation activities and of the research and development sector, respectively, is a key issue for industrialized societies. So far it is rather unclear how economic growth, innovation and factor income shares are linked with each other although standard concepts of production functions lend themselves as a natural starting point. The book of Thomas Piketty on Capitalism in the 21st Century has raised new interest in these issues, particularly since Piketty provides new historical statistics on medium and long-term changes in the capital income share in industrialized countries. The subsequent contribution sheds new theoretical light on the issues above.

Modern growth theory has brought interesting insights into the nature of innovation and growth; with innovation often associated with a rise in the number of product varieties that are used as intermediate products. Modern growth approaches are largely organized within the framework of complex growth models on the basis of a very specific utility function (e.g. AGHION/HOWITT, 2009), namely infinitely-lived households with a rate of time preference $\rho$ discount factor $V^{\prime}=1 /(1+\rho)$ - and an isoelastic utility function where utility $U$ depends on consumption C: Hence the function is $U(C)=(\exp (1-\varepsilon)-1) /(1-\varepsilon)$; here the crucial intertemporal elasticity of substitution is $\eta:=1 / \varepsilon>0$ where (with $t$ denoting the time index) the relevant Euler equation becomes $-\varepsilon(\mathrm{dC} / \mathrm{dt}) / \mathrm{C}=\rho-\mathrm{r}$ ( $\mathrm{r}$ is the real interest rate); or equivalently, if $\mathrm{C}(\mathrm{t})$ is growing at the constant rate $g$ we have real interest rate $r=\rho+\varepsilon g$ : The equilibrium real interest rate must increase by $\varepsilon$ percentage points for a one percentage point rise in the growth rate; or the equation can be restated as $\mathrm{g}=(\mathrm{r}-\rho) \eta$; in the context of a ROMER model - with $\lambda$ denoting a productivity parameter in the research sector where product varieties are developed that feed into output (where an output parameter $\alpha^{\prime \prime}>0$; L is the size the workforce) one gets for the growth rate $(\mathrm{g})$ the expression $\mathrm{g}=\left(\alpha^{\prime \prime} \lambda \mathrm{L}-\rho\right) /\left(\alpha^{\prime \prime}+\varepsilon\right)$ (for a summary analysis see AGHION/HOWITT, 2009, pp. 74-76). In this approach, the size of the respective country, as proxied by the labor force $\mathrm{L}$, plays a role for the growth rate, which is not very plausible in view of empirical findings (JONES, 1995); and the recent debate about key macroeconomic issues also raises new questions (WELFENS, 2014).

The more traditional neoclassical growth model, however, has been rather neglected, although it can still be a very useful workhorse for important analytical issues (WELFENS, 2011); a modified growth model of SOLOW (1957) on the basis of a Cobb-Douglas production function and a particular new progress function will subsequently be combined with a new bias in technology that might be of particular relevance to a modern reality that has been characterized by a rising share of capital income in OECD countries (PIKETTY, 2014).

In his book "Capitalism in the 21st Century", Thomas Piketty has emphasized that the CobbDouglas production function has become popular in Economics text books; the basic version of that function is $Y=K^{\beta} L^{(1-\beta)}$ where $Y$ is output, $K$ capital and $L$ labor, while $\beta$ is the output elasticity of capital; it can be shown that under competition in goods and factor markets $\beta(0<\beta<1)$ is equal to the income share of capital while $1-\beta$ is, of course, the income share of labor. In principle the exponent for labor could be smaller or larger than 1- $\beta$, but then the income shares of both input factors would no longer sum up to unity which is inconsistent unless we give the 
assumptions of competition in goods markets and labor markets plus profit maximization. The CES production function is, of course, more variable in terms of factor income results (and also is more useful than the CD function when it comes to empirical implementation in the context of an augmented function with knowledge as an input), but it is also a bit more complex. Moreover, one can consider a wider range of input factors, e.g. in addition to $\mathrm{K}$ and $\mathrm{L}$ one may consider energy E, knowledge A or land V; Piketty's analysis, for example, has emphasized the role of land in a historical perspective of the 19th century. With respect to France, Piketty shows (Figure 6.8) that the capital share in national income in 1900-2010 has considerably reduced in the 1910s, in the 1930s - after the Great Depression - and in the 1940s. In 1950 the share had recovered and slightly exceeded $25 \%$, but it decreased in the decade after the oil price shock of the 1970s and then increased again in the period 1990-2010 - in the beginning of the digital economy age when it reached a peak of almost 30 percent.

Piketty writes under the implicit assumption that the output elasticity of a Cobb-Douglas production function is constant (pp. 224-225): "I have just shown that the Cobb-Douglas hypothesis of a completely stable capital-labor split cannot give a totally satisfactory explanation of the long-term evolution of the capital-labor split...The most important case, which I discussed briefly in the Introduction, is no doubt the increase in capital's share of income during the early phase of the Industrial Revolution, from 1800 to 1860. In Britain, for which we have the most complete data, the available historical studies, in particular those of Robert Allen...suggest that capital's share increased by something like 10 percent of national income, from 35-40 percent in the late eighteenth and early nineteenth centuries to around 45-50 percent in the middle of the nineteenth century, when Marx wrote the Communist Manifesto and set to work on by a comparable decrease in capital's share in the period 1870-1900, followed by a slight increase between 1900 and 1910, so that in the end the capital share was probably not very different around the turn of the twentieth century from what it was during the French Revolution and Napoleonic area...We therefore can speak of a "medium-term" movement rather than a durable long-term trend. Nevertheless, this transfer of 10 percent of national income to capital during the first half of the nineteenth century was by no means negligible...According to Allen, the main explanation for this was the exodus of labor from the countryside and into the cities, together with technological changes that increased the productivity of capital (reflected by a structural change in the production function) - the caprices of technology, in short."

Indeed the following new approach, with a more flexible Cobb-Douglas production function whose output elasticity of capital is a function of the size of the R\&D sector, will allow for structural changes in the production function and thus one can test to what extent the R\&D activities and innovation dynamics, respectively, cause a bias in favor of capital income. From a theoretical perspective, one may argue that the goodwill of firms, being part of capital broadly defined, should indeed reflect the innovation performance and reputation of the respective firm, respectively: if the relative size of the R\&D sector is rising, the goodwill of firms should increase, the immaterial capital - in real terms - will thus increase which amounts to saying that there is Solow-neutral technological progress; alternatively, one could argue that the output elasticity of physical capital has increased, namely to the extent that a higher goodwill indicates that the existing physical stock of capital is used in a more productive way; and with the output elasticity of the capital stock rising the marginal product of capital $\mathrm{K}$ is increasing.

As major changes in the capital income share occur in both the medium term and the long run, it is adequate to analyze such changes in the context of an adequate growth modeling. Modern endogenous growth approaches offer interesting options to generate sustained growth in a 
model. ROMER (1987), for example, has developed a growth model with expanding variety, where the rise of varieties on the input side avoids that decreasing returns stop output growth. ROMER (1990) has extended the product-variety approach by additionally considering an R\&D sector whose task is to create blueprints for new inputs as a consequence of innovations so that technological progress is endogenized in combination with profitable R\&D and variety expansion. The role of regional market integration and growth was analyzed by GROSSMAN/HELPMAN (1991) and RIVERA-BATIZ/ROMER (1991). The subsequent technological progress function - picking up the approach of KALDOR (1957) - is silent on the exact mechanism of knowledge generation, however, in principle, the parameters used in the equation could, under certain conditions, be traced to existing innovation approaches and endogenous growth models, respectively. Thus the exact mechanism of knowledge growth is not a major focus of this contribution, rather there is the modest goal to combine the emergence of an R\&D sector - a strong phenomenon of the Industrial Revolution - and changes in the share of capital income and growth, respectively. Moreover, an important normative issue is picked up, namely, to what extent government can choose the size of the R\&D sector in a way that per capita consumption is maximized in the steady state: the golden rule analysis is thus enriched by a new aspect.

Traditionally, technological progress has been classified in various ways, for example embodied vs. disembodied, Solow-neutral, Hicks-neutral or Harrod-neutral, the latter will to some extent be picked up here: Knowledge expansion occurs in such a way that it amounts to an effective rise of labor input (Solow neutrality, by contrast, means that technological progress is capital enhancing and Hicks neutrality means that the expansion of knowledge (A) amounts to a proportionate rise of both labor (L) and capital (K). The subsequent bias considered is such that $R \& D$ activities raise knowledge in a labor-augmenting way, while at the same time increasing the output elasticity of capital and reducing the output elasticity of labor; in the context of the new Cobb-Douglas function suggested, this implies - assuming competition in labor and goods markets - that the income share of capital will increase and that of labor reduce. In the context of both a closed economy and an open economy there are further important implications.

The modifications suggested to the traditional growth analysis are rather modest at first glance, but there are powerful implications. The progress function used is not derived from a microeconomic optimization calculus, but it is obvisouly in line with some stylized facts observed in industrialized countries, including newly industrialized countries. It should be emphasized at this point that research \& development activities of firms are considered here as an intermediate input, not as final output (as in the recent regime change of the UN System of National Accounts).

A key insight from the simple new model is that one can determine the golden rule in a new way - the rule had initially been established by PHELPS (1961) and VON WEIZSÄCKER (1962) and indicates a steady state of per-capita-capital accumulation which will bring about a maximization of consumption per capita in a closed economy.

\section{Stylized Facts: Capital Income, Gini Coefficients, R\&D-GDP Ratios and Technological Progress Rate}

The following tables show that international developments are by no way uniform in the critical fields of income inequality and innovation dynamics. One can, however, not overlook the fact that the Gini coefficient in the US has clearly increased over the long run. As regards the role of 
R\&D services imported from abroad, France and Japan, as well as Slovenia, show a strong decline between the mid-1990s and the mid-2000s, while Finland indicates a strong increase that may be interpreted as the ability to absorb global technological progress rather effectively. The same applies to the US, Norway, Poland and Korea. Germany and the Netherlands stand for a rather stable performance in this respect. One may emphasize that there is technoglobalization - rising internationalization of the R\&D process in leading firms - over the long run, but there is a stagnation of that process in the first decade of the twenty first century (LAURENS ET AL., 2015; JUNGMITTAG, 2015).

As regards the capital income share between 1995 and 2005, it has increased in many countries (Tab. 1); it is very high in Mexico, but for this particular finding several country-specific elements are likely to play a role. In Greece, as in the Czech Republic and Lithuania, the income share of capital has declined, where economic opening up in eastern Europe and the effects of EU membership - bringing more competition - might have contributed to that development in the two eastern European countries. To what extent more innovation could explain the rise of the capital income share observed in most countries is unclear and so far there is no analytical framework for the necessary empirical analysis. It should be emphasized that the capital income share can change if the structure of the population is changing: e.g. if the number of selfemployed farmers declines over time - with most former farmers finding a new job as an employee or worker - the share of capital income will decline for structural reasons; figures in Table 1 do not take account of this, howevr it is well known from figures, for example in relation to for Germany or France, that such long run structural effects should not be neglected in empirical analysis. Nevertheless, the capital income share is rising for most countries shown and there also is a rise of the Gini coefficient (Tab. 2).

As regards technological progress, there are some indications from input output analysis that internationalization of the $R \& D$ process plays a role (Tab. 3) as there is a rise of imported $R \& D$ services; here Italy is almost an outlier - the share of R\&D services imported to Italy has declined over time and this - along with a very low ratio of foreign direct investment inflows relative to GDP - could be part and parcel of the weak growth performance of the country. The additional tables all indicate the rising role of research and development in industrialized countries and many newly industrialized countries. With respect to technological progress as covered by total sector productivity growth (Tab. 7), there are considerable international differences - part of such differences should obviously be explained by cross-country differences in R\&D activities and innovation efficiency (the latter partly related to the national innovation system). 
Table 1: $\quad$ Capital income share $^{1)}$ as a \% of GDP

\begin{tabular}{|c|c|c|c|c|}
\hline Country & 1995 & 2000 & 2005 & Change $(\Delta)$ \\
\hline Belgium & $39 \%$ & $40 \%$ & $41 \%$ & $3 \%$ \\
\hline Bulgaria & $49 \%$ & $51 \%$ & $53 \%$ & $4 \%$ \\
\hline Czech Republic & $56 \%$ & $55 \%$ & $53 \%$ & $-2 \%$ \\
\hline Denmark & $45 \%$ & $46 \%$ & $46 \%$ & $0 \%$ \\
\hline Germany & $41 \%$ & $41 \%$ & $44 \%$ & $3 \%$ \\
\hline Estonia & $44 \%$ & $51 \%$ & $52 \%$ & $8 \%$ \\
\hline Ireland & $45 \%$ & $53 \%$ & $53 \%$ & $8 \%$ \\
\hline Greece & $51 \%$ & $50 \%$ & $46 \%$ & $-5 \%$ \\
\hline Spain & $40 \%$ & $42 \%$ & $44 \%$ & $4 \%$ \\
\hline France & $43 \%$ & $44 \%$ & $44 \%$ & $1 \%$ \\
\hline Croatia & NA & $39 \%$ & $43 \%$ & NA \\
\hline Italy & $47 \%$ & $49 \%$ & $48 \%$ & $1 \%$ \\
\hline Cyprus & $47 \%$ & $48 \%$ & $47 \%$ & $0 \%$ \\
\hline Latvia & $52 \%$ & $51 \%$ & $55 \%$ & $3 \%$ \\
\hline Lithuania & $54 \%$ & $51 \%$ & $51 \%$ & $-3 \%$ \\
\hline Luxembourg & $49 \%$ & $51 \%$ & $48 \%$ & $-2 \%$ \\
\hline Hungary & $45 \%$ & $47 \%$ & $47 \%$ & $3 \%$ \\
\hline Malta & $46 \%$ & $51 \%$ & $51 \%$ & $5 \%$ \\
\hline Netherlands & $38 \%$ & $41 \%$ & $42 \%$ & $4 \%$ \\
\hline Austria & $41 \%$ & $44 \%$ & $46 \%$ & $6 \%$ \\
\hline Poland & $42 \%$ & $43 \%$ & $50 \%$ & $9 \%$ \\
\hline Portugal & $41 \%$ & $40 \%$ & $41 \%$ & $0 \%$ \\
\hline Romania & $36 \%$ & $28 \%$ & $41 \%$ & $6 \%$ \\
\hline Slovenia & $32 \%$ & $38 \%$ & $40 \%$ & $7 \%$ \\
\hline Slovakia & $57 \%$ & $55 \%$ & $58 \%$ & $0 \%$ \\
\hline Finland & $44 \%$ & $47 \%$ & $47 \%$ & $3 \%$ \\
\hline Sweden & $53 \%$ & $52 \%$ & $52 \%$ & $0 \%$ \\
\hline United Kingdom & $43 \%$ & $41 \%$ & $41 \%$ & $-2 \%$ \\
\hline Iceland & NA & $35 \%$ & $36 \%$ & NA \\
\hline Norway & $50 \%$ & $54 \%$ & $56 \%$ & $6 \%$ \\
\hline Switzerland & $34 \%$ & $35 \%$ & $35 \%$ & $2 \%$ \\
\hline United States & $40 \%$ & $38 \%$ & $42 \%$ & $1 \%$ \\
\hline Japan & $33 \%$ & $36 \%$ & $40 \%$ & $6 \%$ \\
\hline Canada & $43 \%$ & $44 \%$ & $46 \%$ & $2 \%$ \\
\hline Mexico & $58 \%$ & $59 \%$ & $60 \%$ & $2 \%$ \\
\hline Australia & $42 \%$ & $43 \%$ & $45 \%$ & $3 \%$ \\
\hline New Zealand & $54 \%$ & $56 \%$ & $53 \%$ & $-1 \%$ \\
\hline
\end{tabular}

1) Capital share is calculated as 1-wage income share (Compensation per employee as percentage of GDP at market prices per person employed)

Source: AMECO Database 
Table 2: Gini Coefficient

\begin{tabular}{|c|c|c|c|c|}
\hline Country & mid 1990s & 2000 & mid 2000s & Change $^{1)}$ \\
\hline Australia & 0.309 & 0.317 & 0.315 & 0.006 \\
\hline Austria & 0.238 & 0.252 & 0.265 & 0.027 \\
\hline Belgium & 0.287 & 0.289 & 0.271 & -0.016 \\
\hline Canada & 0.289 & 0.318 & 0.317 & 0.028 \\
\hline Switzerland & NA & 0.279 & 0.276 & NA \\
\hline Chile & 0.527 & NA & 0.503 & -0.024 \\
\hline Czech Republic & 0.257 & 0.26 & 0.268 & 0.011 \\
\hline Germany & 0.266 & 0.264 & 0.285 & 0.019 \\
\hline Denmark & 0.215 & 0.226 & 0.232 & 0.017 \\
\hline Spain & 0.343 & 0.342 & 0.319 & -0.024 \\
\hline Estonia & NA & NA & 0.349 & NA \\
\hline Finland & 0.228 & 0.261 & 0.269 & 0.041 \\
\hline France & 0.277 & 0.287 & 0.288 & 0.011 \\
\hline United Kingdom & 0.312 & 0.363 & 0.331 & 0.019 \\
\hline Greece & 0.336 & 0.345 & 0.321 & -0.015 \\
\hline Hungary & 0.294 & 0.293 & 0.291 & -0.003 \\
\hline Ireland & 0.324 & 0.304 & 0.314 & -0.01 \\
\hline Israel & 0.338 & 0.347 & 0.378 & 0.04 \\
\hline Italy & 0.348 & 0.343 & 0.352 & 0.004 \\
\hline Japan & 0.323 & 0.337 & 0.321 & -0.002 \\
\hline Korea & NA & NA & 0.306 & NA \\
\hline Luxembourg & 0.259 & 0.261 & 0.258 & -0.001 \\
\hline Mexico & 0.519 & 0.507 & 0.474 & -0.045 \\
\hline Netherlands & 0.297 & 0.292 & 0.284 & -0.013 \\
\hline Norway & 0.243 & 0.261 & 0.276 & 0.033 \\
\hline New Zealand & 0.335 & 0.339 & 0.335 & 0 \\
\hline Poland & NA & 0.316 & 0.349 & NA \\
\hline Portugal & 0.359 & 0.356 & 0.385 & 0.026 \\
\hline Slovak Republic & NA & NA & 0.268 & NA \\
\hline Slovenia & NA & NA & 0.246 & NA \\
\hline Sweden & 0.211 & 0.243 & 0.234 & 0.023 \\
\hline Turkey & 0.49 & NA & 0.43 & -0.06 \\
\hline United States & 0.361 & 0.357 & 0.38 & 0.019 \\
\hline
\end{tabular}

1) mid $2000 \mathrm{~s}$ relative to mid $1990 \mathrm{~s}$

Source: OECD Factbook 2011-2012 
Table 3: Imported R\&D services from abroad which is used as intermediate input (as a \% of GDP/total value added)

\begin{tabular}{|c|c|c|c|}
\hline Country & mid 1990s* & early 2000s* & mid 2000s* \\
\hline Australia & $0.000 \%$ & $0.000 \%$ & $0.000 \%$ \\
\hline Austria & $0.101 \%$ & $0.176 \%$ & $0.109 \%$ \\
\hline Belgium & $0.208 \%$ & $0.280 \%$ & $0.433 \%$ \\
\hline Canada & $0.000 \%$ & $0.000 \%$ & $0.000 \%$ \\
\hline Chile & $0.000 \%$ & NA & $0.000 \%$ \\
\hline Czech Republic & $0.012 \%$ & $0.207 \%$ & $0.164 \%$ \\
\hline Denmark & $0.000 \%$ & $0.084 \%$ & $0.258 \%$ \\
\hline Estonia & $0.000 \%$ & $0.000 \%$ & $0.016 \%$ \\
\hline Finland & $0.126 \%$ & $0.112 \%$ & $1.297 \%$ \\
\hline France & $0.095 \%$ & $0.088 \%$ & $0.106 \%$ \\
\hline Germany & $0.137 \%$ & $0.239 \%$ & $0.230 \%$ \\
\hline Greece & $0.000 \%$ & $0.011 \%$ & $0.024 \%$ \\
\hline Hungary & $0.000 \%$ & $0.000 \%$ & $0.277 \%$ \\
\hline Ireland & $0.000 \%$ & $2.452 \%$ & $2.676 \%$ \\
\hline Israel & $0.000 \%$ & NA & $0.000 \%$ \\
\hline Italy & $0.043 \%$ & $0.033 \%$ & $0.028 \%$ \\
\hline Japan & $0.006 \%$ & $0.008 \%$ & $0.005 \%$ \\
\hline Korea & NA & $0.000 \%$ & $0.278 \%$ \\
\hline Luxembourg & $0.328 \%$ & $0.184 \%$ & $0.072 \%$ \\
\hline Mexico & NA & NA & $0.000 \%$ \\
\hline Netherlands & $0.380 \%$ & $0.503 \%$ & $0.790 \%$ \\
\hline New Zealand & $0.013 \%$ & $0.000 \%$ & NA \\
\hline Norway & $0.081 \%$ & $0.083 \%$ & $0.083 \%$ \\
\hline Poland & $0.023 \%$ & $0.025 \%$ & $0.040 \%$ \\
\hline Portugal & $0.018 \%$ & $0.014 \%$ & $0.019 \%$ \\
\hline Slovak Republic & $0.102 \%$ & $0.034 \%$ & $0.074 \%$ \\
\hline Slovenia & $0.135 \%$ & $0.155 \%$ & $0.157 \%$ \\
\hline Spain & $0.027 \%$ & $0.049 \%$ & $0.076 \%$ \\
\hline Sweden & $0.164 \%$ & $0.607 \%$ & $0.000 \%$ \\
\hline Switzerland & NA & $0.000 \%$ & $0.178 \%$ \\
\hline Turkey & $0.000 \%$ & $0.000 \%$ & $0.000 \%$ \\
\hline United Kingdom & $0.100 \%$ & $0.084 \%$ & $0.181 \%$ \\
\hline United States & $0.005 \%$ & $0.052 \%$ & $0.071 \%$ \\
\hline
\end{tabular}

Source: OECD STAN IO Database 
Table 4: $\quad$ Total R\&D services which is used as intermediate input (as a \% of GDP/total value added)

\begin{tabular}{|c|c|c|c|}
\hline Country & mid 1990s & early 2000s & mid 2000s \\
\hline Australia & $0.000 \%$ & $0.000 \%$ & $0.000 \%$ \\
\hline Austria & $0.207 \%$ & $0.262 \%$ & $0.222 \%$ \\
\hline Belgium & $0.217 \%$ & $0.342 \%$ & $0.556 \%$ \\
\hline Canada & $0.000 \%$ & $0.000 \%$ & $0.000 \%$ \\
\hline Chile & $0.000 \%$ & NA & $0.000 \%$ \\
\hline Czech Republic & $0.365 \%$ & $0.519 \%$ & $0.456 \%$ \\
\hline Denmark & $0.157 \%$ & $0.307 \%$ & $0.484 \%$ \\
\hline Estonia & $0.107 \%$ & $0.108 \%$ & $0.124 \%$ \\
\hline Finland & $0.338 \%$ & $0.280 \%$ & $1.338 \%$ \\
\hline France & $1.648 \%$ & $1.629 \%$ & $1.541 \%$ \\
\hline Germany & $0.268 \%$ & $0.553 \%$ & $0.398 \%$ \\
\hline Greece & $0.059 \%$ & $0.053 \%$ & $0.129 \%$ \\
\hline Hungary & $0.297 \%$ & $0.345 \%$ & $0.561 \%$ \\
\hline Ireland & $0.066 \%$ & $2.532 \%$ & $2.817 \%$ \\
\hline Israel & $0.000 \%$ & NA & $0.000 \%$ \\
\hline Italy & $0.402 \%$ & $0.476 \%$ & $0.491 \%$ \\
\hline Japan & $2.020 \%$ & $2.336 \%$ & $2.515 \%$ \\
\hline Korea & NA & $1.814 \%$ & $2.207 \%$ \\
\hline Luxembourg & $0.428 \%$ & $0.428 \%$ & $0.181 \%$ \\
\hline Mexico & NA & NA & $0.000 \%$ \\
\hline Netherlands & $0.728 \%$ & $0.868 \%$ & $0.997 \%$ \\
\hline New Zealand & $0.273 \%$ & $0.000 \%$ & NA \\
\hline Norway & $0.466 \%$ & $0.582 \%$ & $0.509 \%$ \\
\hline Poland & $0.023 \%$ & $0.321 \%$ & $0.525 \%$ \\
\hline Portugal & $0.122 \%$ & $0.279 \%$ & $0.291 \%$ \\
\hline Slovak Republic & $0.688 \%$ & $0.682 \%$ & $0.337 \%$ \\
\hline Slovenia & $1.058 \%$ & $0.728 \%$ & $0.522 \%$ \\
\hline Spain & $0.062 \%$ & $0.097 \%$ & $0.127 \%$ \\
\hline Sweden & $0.762 \%$ & $1.223 \%$ & $0.000 \%$ \\
\hline Switzerland & NA & $0.665 \%$ & $1.471 \%$ \\
\hline Turkey & $0.063 \%$ & $0.027 \%$ & $0.004 \%$ \\
\hline United Kingdom & $0.656 \%$ & $0.577 \%$ & $0.519 \%$ \\
\hline United States & $0.450 \%$ & $6.203 \%$ & $6.864 \%$ \\
\hline
\end{tabular}

Source: OECD STAN IO Database 
Table 5: $\quad$ Total Domestic $R \& D$ services which is used as intermediate input (as a $\%$ of GDP/total value added)

\begin{tabular}{|c|c|c|c|}
\hline Country & mid 1990s & early 2000s & mid 2000s \\
\hline Australia & $0.000 \%$ & $0.000 \%$ & $0.000 \%$ \\
\hline Austria & $0.105 \%$ & $0.086 \%$ & $0.112 \%$ \\
\hline Belgium & $0.009 \%$ & $0.062 \%$ & $0.124 \%$ \\
\hline Canada & $0.000 \%$ & $0.000 \%$ & $0.000 \%$ \\
\hline Chile & $0.000 \%$ & \#WERT! & $0.000 \%$ \\
\hline Czech Republic & $0.353 \%$ & $0.311 \%$ & $0.293 \%$ \\
\hline Denmark & $0.157 \%$ & $0.222 \%$ & $0.226 \%$ \\
\hline Estonia & $0.107 \%$ & $0.108 \%$ & $0.108 \%$ \\
\hline Finland & $0.212 \%$ & $0.168 \%$ & $0.041 \%$ \\
\hline France & $1.553 \%$ & $1.540 \%$ & $1.436 \%$ \\
\hline Germany & $0.131 \%$ & $0.314 \%$ & $0.168 \%$ \\
\hline Greece & $0.059 \%$ & $0.042 \%$ & $0.105 \%$ \\
\hline Hungary & $0.297 \%$ & $0.345 \%$ & $0.284 \%$ \\
\hline Ireland & $0.066 \%$ & $0.080 \%$ & $0.141 \%$ \\
\hline Israel & $0.000 \%$ & $\#$ & $0.000 \%$ \\
\hline Italy & $0.359 \%$ & $0.443 \%$ & $0.462 \%$ \\
\hline Japan & $2.014 \%$ & $2.327 \%$ & $2.510 \%$ \\
\hline Korea & $\#$ & $1.814 \%$ & $1.929 \%$ \\
\hline Luxembourg & $0.100 \%$ & $0.244 \%$ & $0.110 \%$ \\
\hline Mexico & $\#$ & $\#$ & $0.000 \%$ \\
\hline Netherlands & $0.347 \%$ & $0.364 \%$ & $0.207 \%$ \\
\hline New Zealand & $0.260 \%$ & $0.000 \%$ & $\#$ \\
\hline Norway & $0.386 \%$ & $0.498 \%$ & $0.426 \%$ \\
\hline Poland & $0.000 \%$ & $0.296 \%$ & $0.485 \%$ \\
\hline Portugal & $0.104 \%$ & $0.265 \%$ & $0.271 \%$ \\
\hline Slovak Republic & $0.587 \%$ & $0.649 \%$ & $0.263 \%$ \\
\hline Slovenia & $0.923 \%$ & $0.573 \%$ & $0.365 \%$ \\
\hline Spain & $0.035 \%$ & $0.048 \%$ & $0.050 \%$ \\
\hline Sweden & $0.599 \%$ & $0.615 \%$ & $0.000 \%$ \\
\hline Switzerland & $\#$ & $0.665 \%$ & $1.293 \%$ \\
\hline Turkey & $0.063 \%$ & $0.027 \%$ & $0.004 \%$ \\
\hline United Kingdom & $0.556 \%$ & $0.493 \%$ & $0.338 \%$ \\
\hline United States & $0.445 \%$ & $6.150 \%$ & $6.793 \%$ \\
\hline
\end{tabular}

Source: OECD STAN IO Database 
Table 6: Total intramural R\&D expenditure (GERD) [as a \% of GDP]

\begin{tabular}{|c|c|c|c|}
\hline GEO/TIME & 1995 & 2000 & 2005 \\
\hline Belgium & 1.64 & 1.93 & 1.78 \\
\hline Bulgaria & 0.56 & 0.49 & 0.45 \\
\hline Czech Republic & 0.88 & 1.12 & 1.17 \\
\hline Denmark & 1.79 & 2.19 & 2.39 \\
\hline Germany & 2.13 & 2.4 & 2.43 \\
\hline Estonia & : & 0.6 & 0.92 \\
\hline Ireland & 1.23 & 1.09 & 1.2 \\
\hline Greece & 0.42 & : & 0.58 \\
\hline Spain & 0.77 & 0.89 & 1.1 \\
\hline France & 2.23 & 2.08 & 2.04 \\
\hline Croatia & : & : & 0.86 \\
\hline Italy & 0.94 & 1.01 & 1.05 \\
\hline Cyprus & : & 0.23 & 0.37 \\
\hline Latvia & 0.43 & 0.44 & 0.53 \\
\hline Lithuania & : & : & 0.75 \\
\hline Luxembourg & $:$ & 1.57 & 1.59 \\
\hline Hungary & 0.71 & 0.79 & 0.93 \\
\hline Malta & : & $:$ & 0.53 \\
\hline Netherlands & 1.85 & 1.8 & 1.81 \\
\hline Austria & 1.53 & 1.89 & 2.38 \\
\hline Poland & $:$ & $:$ & 0.57 \\
\hline Portugal & 0.52 & 0.72 & 0.76 \\
\hline Romania & 0.75 & 0.36 & 0.41 \\
\hline Slovenia & 1.49 & 1.36 & 1.41 \\
\hline Slovakia & 0.91 & 0.64 & 0.49 \\
\hline Finland & 2.2 & 3.25 & 3.33 \\
\hline Sweden & 3.13 & : & 3.39 \\
\hline United Kingdom & : & 1.73 & 1.63 \\
\hline Iceland & 1.53 & 2.59 & 2.69 \\
\hline Norway & 1.69 & : & 1.51 \\
\hline Switzerland & : & 2.47 & $:$ \\
\hline Montenegro & $:$ & $:$ & $:$ \\
\hline Serbia & $:$ & $:$ & $:$ \\
\hline Turkey & 0.38 & 0.48 & 0.59 \\
\hline Russia & $:$ & 1.05 & 1.07 \\
\hline United States & 2.4 & 2.62 & 2.51 \\
\hline China (except Hong Kong) & : & $:$ & 1.32 \\
\hline Japan & 2.87 & 3 & 3.31 \\
\hline South Korea & : & 2.3 & 2.79 \\
\hline
\end{tabular}

Source: Eurostat 
Table 7: $\quad$ Total Factor Productivity $(2010=100)$

\begin{tabular}{|c|c|c|c|c|}
\hline Country & 1995 & 2000 & 2005 & $\begin{array}{c}\text { Average annual } \\
\text { growth (1996-2005) }\end{array}$ \\
\hline Latvia & 54,4 & 80,76 & 106,29 & $6.70 \%$ \\
\hline Lithuania & 66,04 & 80,53 & 103,07 & $4.45 \%$ \\
\hline Romania & 70,07 & 73,72 & 103,25 & $3.88 \%$ \\
\hline Estonia & 78,81 & 99,11 & 112,25 & $3.54 \%$ \\
\hline Poland & 69,99 & 83,94 & 93,52 & $2.90 \%$ \\
\hline Ireland & 82,52 & 101,31 & 105,12 & $2.42 \%$ \\
\hline Slovenia & 79,53 & 91,86 & 101,04 & $2.39 \%$ \\
\hline Croatia* & NA & 99,3 & 109,35 & $2.19 \%$ \\
\hline Slovakia & 71,77 & 75,85 & 88,6 & $2.11 \%$ \\
\hline Finland & 82,05 & 95,03 & 101,19 & $2.10 \%$ \\
\hline Sweden & 80,41 & 90,42 & 98,5 & $2.03 \%$ \\
\hline Greece & 87,19 & 97,15 & 106,64 & $2.01 \%$ \\
\hline Iceland** & NA & 93,21 & 102,4 & $1.88 \%$ \\
\hline Hungary & 88,43 & 94,36 & 106,45 & $1.85 \%$ \\
\hline Bulgaria & 89,02 & 94,37 & 105,87 & $1.73 \%$ \\
\hline Czech Republic & 81,72 & 85,18 & 96,4 & $1.65 \%$ \\
\hline United Kingdom & 86,34 & 93,16 & 100,98 & $1.57 \%$ \\
\hline Malta & 89,05 & 100,82 & 102,75 & $1.43 \%$ \\
\hline United States & 84,54 & 92,2 & 97,44 & $1.42 \%$ \\
\hline Norway & 94,99 & 102,22 & 107,85 & $1.27 \%$ \\
\hline Canada & 92,83 & 102,31 & 104,07 & $1.14 \%$ \\
\hline Denmark & 92,43 & 100,4 & 103,37 & $1.12 \%$ \\
\hline Netherlands & 89,21 & 96,27 & 98,92 & $1.03 \%$ \\
\hline Switzerland & 86,79 & 92,46 & 96,05 & $1.01 \%$ \\
\hline Austria & 89,67 & 95,86 & 99,1 & $1.00 \%$ \\
\hline Australia & 93,68 & 100,82 & 103,08 & $0.96 \%$ \\
\hline Belgium & 91,21 & 97,21 & 100,26 & $0.95 \%$ \\
\hline France & 92,31 & 98,53 & 100,65 & $0.86 \%$ \\
\hline New Zealand & 94,86 & 99,82 & 102,95 & $0.82 \%$ \\
\hline Luxembourg & 97,51 & 106,61 & 104,69 & $0.71 \%$ \\
\hline Japan & 90,88 & 92,88 & 97,5 & $0.70 \%$ \\
\hline Cyprus & 99,87 & 106,1 & 105,72 & $0.57 \%$ \\
\hline Mexico & 103,3 & 113,08 & 107,84 & $0.43 \%$ \\
\hline Germany & 94,06 & 96,36 & 97,99 & $0.41 \%$ \\
\hline Portugal & 94,25 & 99,41 & 98,04 & $0.39 \%$ \\
\hline Italy & 100,66 & 105,1 & 103,52 & $0.28 \%$ \\
\hline Spain & 99,98 & 101,91 & 100,59 & $0.06 \%$ \\
\hline
\end{tabular}

Note: * average annual growth 1997-2005, ** average annual growth 2000-2005

Source: AMECO Database 
The links between innovation dynamics - and R\&D activities on the input side - and economic growth and factor income shares should be explained in an adequate analytical framework. In the subsequent analysis, the new model is presented first, while the final section offers some important policy conclusions and perspectives for further research.

\section{New Quasi-Endogenous Growth Model With Biased Technological Progress}

The subsequent approach will consider a model in which part of workers are active in the research and development sector (R\&D) and thus contribute to raising the growth rate of knowledge. It is, however, useful to first establish a simple analytical benchmark.

Thus let us start the analysis with an economy without any technological progress and capital depreciation rate $\delta$; output $Y$ is given in the subsequent full employment model by the following production function (with $0<\beta<1$; for the income tax rate $\tau$ it holds $0<\tau<1$ ):

$$
Y=K^{\beta}(A L)^{(1-\beta)}
$$

Labor is assumed to be given, knowledge growth at a constant exogenous growth rate (a) and the savings function is $S=s(1-\tau) Y$ so that imposing the equilibrium condition for the goods market $S /(\mathrm{AL})=((\mathrm{dK} / \mathrm{dt})+\delta \mathrm{K}) /(\mathrm{AL})$ yields the steady state value for the capital stock per unit of labor in efficiency unity $\left(\mathrm{k}^{\prime}:=\mathrm{K} /(\mathrm{AL})\right)$ :

$$
\mathrm{k}^{\prime} \#=(\mathrm{s}(1-\tau) /(\mathrm{a}+\delta))^{1 /(1-\beta)}
$$

This serves as a useful benchmark in the subsequent analysis (\# denotes the steady state).

Next let us modify the analysis by considering a technological progress function that first was suggested by KALDOR (1957) as a simple concept for analyzing the expansion of knowledge over time. The function suggested subsequently is straightforward as it is assumed that the growth rate of knowledge (a) is enhanced by the share of workers ( $\left.\beta^{\prime}\right)$ working in R\&D firms, at the same time the hypothesis is stated that $\beta^{\prime}>0$ raises the output elasticity of capital so that the new elasticity is $\beta+\beta^{\prime \prime} \beta$ (with $\beta^{\prime \prime}>0$ ) and hence the output elasticity of labor - and the respective income share - is reduced to $1-\beta-\beta^{\prime \prime} \beta^{\prime}$; it will be assumed that $0<\beta+\beta^{\prime \prime} \beta^{\prime}<1$. The new production function - assuming that a share of $\beta^{\prime}$ workers is used in R\&D activities - thus is given by:

$$
Y=K^{\beta+\beta^{\prime \prime} \beta^{\prime}} \cdot\left(A\left(1-\beta^{\prime}\right) L\right)^{1-\beta-\beta^{\prime \prime} \beta^{\prime}}
$$




$$
Y=\left(1-\beta^{\prime}\right)^{1-\beta-\beta^{\prime \prime} \beta^{\prime}} \cdot K^{\beta+\beta^{\prime \prime} \beta^{\prime}} \cdot(A L)^{1-\beta-\beta^{\prime \prime} \beta^{\prime}}
$$

An obvious implication is that the income share of capital will rise in a competitive setting, namely with goods and factor market in equilibrium, so that profit maximization implies that the income share of capital is equal to the output elasticity $\beta+\beta$ " $\beta$. This could be a new explanation for the rise of inequality in industrialized countries as emphasized by PIKETTY (2014) and others (incidentally, one could also consider that part of the capital stock is used for R\&D activities which then stands for a different new bias in technology).

The simple progress function suggested here assumes that there is a depreciation rate $\lambda^{\prime}$ while the rise of the progress rate is described by a term in which $\beta^{\prime}$ appears, as well as an effectiveness parameter $\lambda^{\prime}$ and an innovation efficiency parameter $\mathrm{v}$; note that in an open economy $\lambda^{\prime}$ might be decomposed into a domestic parater $\lambda$ plus an additional term $\alpha^{\prime} \alpha^{*}$ where $\alpha^{*}$ is the share of capital owned by foreign investors (the parameter $\alpha^{\prime}>0$ ), but in the presence of cumulated FDI inflows the savings function has to be adjusted (see WELFENS, 2011). In the simple economy considered here, the progress function is stated as follows (with $0<v<1, \lambda^{\prime}>0$, $\lambda$ ">0; $\mathrm{t}$ is the time index):

$$
\frac{d a}{d t}=\lambda^{\prime \prime} \beta^{\prime} a^{v}-\lambda^{\prime} a
$$

The solution of this Bernoullian differential equation is given by the following term for the steady state value a\#:

$$
a \#=\left(\frac{\lambda^{\prime \prime} \beta^{\prime}}{\lambda^{\prime}}\right)^{\frac{1}{(1-v)}}
$$

Here it will be assumed that the convergence to the steady state value is sufficiently high so that we can asymptotically use our (modified) standard steady state solution for the differential equation for $k^{\prime}$, namely $k^{\prime} \#=\left[\left(s(1-\tau)\left(1-\beta^{\prime}\right)^{1-\beta-\beta-\beta^{\prime \prime} \beta^{\prime}} /(a+\delta)\right]^{1 /\left(1-\beta-\beta^{\prime \prime} \beta^{\prime}\right)}\right.$. The traditional differential equation with a savings function $S=s(1-\tau) Y$ can be stated for $k^{\prime}:=K /(A L)$ - where AL is labor in efficiency units $-a s d k^{\prime} / d t=s(1-\tau) k^{\prime} \beta-(a+\delta) k^{\prime}$. In the new setup the exponent for $k^{\prime}$ is greater than $B$ and there is an additional term in the savings function which indicates that part of labor is devoted not to the production of final output, rather a share of workers $\beta^{\prime}$ is used to conduct $\mathrm{R} \& \mathrm{D}$.

If the steady state solution is to result in a maximization of per capita income $(C / L)$ and $C /(A L)$, respectively, the standard golden age condition requires that the marginal product of capital be equal to $a+\delta$. However, in the new model setup we have $S=s(1-\tau) Y$. Maximization of $C /(A L)$ in the steady state requires one to consider $C /(A L)=y^{\prime}\left(k^{\prime}\right)-(a+\delta) k^{\prime}-G /(A L)$; we can replace $\mathrm{G} /(\mathrm{AL})$ by $\gamma \mathrm{y}$, where $\gamma:=\mathrm{G} / \mathrm{Y}$. Therefore $\mathrm{C} /(\mathrm{AL})=(1-\tau) \mathrm{y}^{\prime}\left(\mathrm{k}^{\prime}\right)-(\mathrm{a}+\delta) \mathrm{k}^{\prime}$; a balanced budget has been assumed here so that $\gamma=\tau$. Hence one must have in the new model setup that (1- 


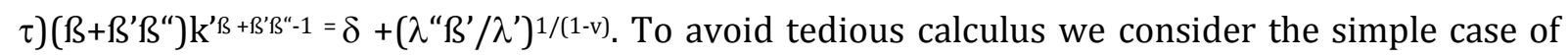
$\delta=0$ so that we have:

$$
(1-\tau)\left(\beta+\beta^{\prime \prime} \beta^{\prime}\right) k^{\prime \beta+\beta^{\prime \prime} \beta^{\prime-1}}=\left(\frac{\lambda^{\prime \prime} \beta^{\prime}}{\lambda^{\prime}}\right)^{\frac{1}{(1-v)}}
$$

Assuming $\tau$ to be small so that $\ln (1-\tau) \approx-\tau$ we get:

$$
-\tau+\ln \left(\beta+\beta^{\prime \prime} \beta^{\prime}\right)-\left(1-\beta-\beta^{\prime \prime} \beta^{\prime}\right) \ln k^{\prime}=\left(\frac{1}{(1-v)}\right)\left(\ln \lambda^{\prime \prime}+\ln \beta^{\prime}-\ln \lambda^{\prime}\right)
$$

(7)

$$
\ln k^{\prime}=\frac{-\tau+\ln \left(\beta+\beta^{\prime \prime} \beta^{\prime}\right)-\left(\frac{1}{(1-v)}\right)\left(\ln \lambda^{\prime \prime}+\ln \beta^{\prime}-\ln \lambda^{\prime}\right)}{\left(1-\beta-\beta^{\prime \prime} \beta^{\prime}\right)}
$$

For lnk'\# and k'\#, respectively, we have the analogy to the traditional steady state solution:

(8) $\quad k^{\prime} \#=\left(\frac{s(1-\tau)\left(1-\beta^{\prime}\right)^{1-\beta-\beta^{\prime \prime} \beta^{\prime}}}{a}\right)^{\frac{1}{\left(1-\beta-\beta^{\prime \prime} \beta^{\prime}\right)}}$

Taking logs, while taking into account the approximization $\ln \left(1-\beta^{\prime}\right) \approx-\beta^{\prime}$ and $\ln (1-\tau) \approx-\tau$ and the equation (4) for a\#, we get:

$$
\ln k^{\prime} \#=-\beta^{\prime}+\left(\frac{1}{\left(1-\beta-\beta^{\prime \prime} \beta^{\prime}\right)}\right)\left(\ln s-\tau-\left(\frac{1}{(1-v)}\right)\left(\ln \lambda^{\prime \prime}+\ln \beta^{\prime}-\ln \lambda^{\prime}\right)\right)
$$

Inserting $\mathrm{k}^{\prime}$ and $\operatorname{lnk}^{\prime} \#$, respectively (from equation (7)), one may now determine the optimum size of the R\&D sector, namely determine $\beta^{\prime}$ which maximizes $C / L$ and $C /(A L)$, respectively:

$$
-\beta^{\prime}\left(1-\beta-\beta^{\prime \prime} \beta^{\prime}\right)-\ln \left(\beta+\beta^{\prime \prime} \beta^{\prime}\right)+\tau=\tau-\ln s
$$

Thus we have an implicit solution for the optimum size of the R\&D sector which reflects an interesting trade-off: 
- The higher $\beta^{\prime}$ is, the lower the production of current real output is - as less workers are employed in production of final output.

- The higher $\AA^{\prime}$ is, the higher the progress rate in the long run is; in the steady state (with $A_{0}$ standing for the initial level of knowledge; $e^{\prime}$ is the Euler number) we have $A(t)=A_{0} \exp \left[\left(\lambda^{\prime \prime} \beta^{\prime} / \lambda^{\prime}\right)^{1 /(1-v)} t\right]$ and therefore: $\ln A(t)=\ln A_{0}+\left(\lambda^{\prime \prime} \beta^{\prime} / \lambda^{\prime}\right)^{1 /(1-v) t}$.

Thus, an economy which switches from being an economy with no R\&D sector and zero technological progress towards an economy with an R\&D sector will experience an instantaneous initial decline of output - as part of the labor force shifts to the new R\&D sector but will face a higher growth rate of output in the steady state. If politicians and voters, respectively, are not extremely myopic, then the opportunity to introduce an R\&D sector will be realized by the political system (this conjecture does not mean to overlook the reality that the R\&D sector will need skilled workers and education investment, respectively, and skilled workers might be less likely than unskilled workers to accept an authoritarian system so that certain political systems might indeed shy away from innovation - only to find out that competing societies, with a rapid expansion of knowledge, will start to dominate the backward economy and political system in due time).

The optimal $\beta^{\prime}$ is given by the expression (for derivation see appendix):

$$
\beta^{\prime}=\frac{1}{2 \beta \beta^{\prime \prime}}\left[\left((1-\beta) \beta+\beta^{\prime \prime}\right)+\sqrt{\left((1-\beta) \beta+\beta^{\prime \prime}\right)^{2}+4 \beta^{\prime \prime} \beta^{2}(\ln (s)+\ln (\beta))}\right]
$$

The optimum $\beta^{\prime}$ is the negative function of the savings function and not a function of the income tax rate. The impact of the R\&D efficiency parameter $\beta^{\prime \prime}$ is ambiguous. This holds particularly if one would additionally take into account that government $R \& D$ promotion programmes in reality are typically linked to $\Omega^{\prime \prime}$, so that via the government budget constraint indeed the income tax rate is indeed also linked to $\beta^{\prime \prime}$ (or possibly $\beta^{\prime \prime 2}$ ).

As regards the overall economic significance of $\beta^{\prime}$, it is fairly obvious that the steady state solution for $y^{\prime}$ indeed implies an interesting trade-off with respect to the impact of $\beta^{\prime}$ on the level of the growth path (where we take logs, while taking into account that $y^{\prime}:=Y /(A L)=k^{\prime} \beta+\beta^{\prime} \beta^{\prime \prime} ;$ the approximization $\ln \left(1-\beta^{\prime}\right) \approx-\beta^{\prime}$ and $\ln (1-\tau) \approx \tau$ is used; $\left.v^{\prime \prime}:=1 /(1-v)\right)$ :

$$
\ln y^{\prime} \#=-\beta^{\prime}+\left(\left(ß+\beta^{\prime \prime} ß\right) /\left(1-\beta^{\prime \prime} \beta^{\prime} \beta^{\prime}\right)\right)\left[\ln s-\tau-v^{\prime \prime}\left(\ln \lambda^{\prime \prime}+\ln \beta^{\prime}-\ln \lambda\right)\right]
$$

The squared-bracked term is positive (assuming that $\left.y^{\prime} \#>1\right)$ and the term $\left(\left(\beta+\beta^{\prime \prime} \beta\right) /\left(1-\beta-\beta^{\prime \prime} \beta^{\prime}\right)\right.$ can be rewritten as $1 /((1 / \sigma)-1)$ where $\sigma:=\beta+\beta^{\prime \prime} \beta^{\prime}$ so that $\beta^{\prime}$ has a negative impact via the first right-hand term $-\beta^{\prime}$ (the fact that part of workers are active in R\&D) and via $-v^{\prime \prime} \mid n \beta^{\prime}$. The third element containing $\aleph^{\prime}$ has a positive impact on lny' so that there is a true trade-off of the size of the R\&D sector. As regards changes in the capital income share, the two additional elements $\Omega^{\prime \prime}$ and $\beta^{\prime}$ ' stand for two new potential impulses explaining a share of capital income over time. Here empirical research is needed. This basic idea could, of course, also be implemented in a CES production function. Whether or not the expansion of the ICT sector is a major driver that has 
raised the parameter $\beta^{\prime \prime}$ and $\beta^{\prime}$ - or reduced $\mathrm{v}$ - is of particular interest for future empirical research.

If one takes a look at lny ( $y$ is per captia income), the initial development of the economy would be described by the line $\mathrm{ABC}$ (Fig. 1). If in $\mathrm{t}^{\prime}$ a rise of the R\&D sector occurs, the level of the growth path will decline (see point B') while the new growth rate of per capita income will increase as shown in the line B'DE. In welfare analysis, there will be some point in time $t^{\prime \prime}$ at which the discounted income gain from higher growth has exceeded the transitory decline of per capita income that has occurred at point $\mathrm{t}^{\prime}$.

\section{Figure 1: Rise of the R\&D Sector in the Quasi-Neoclassical Growth Model}

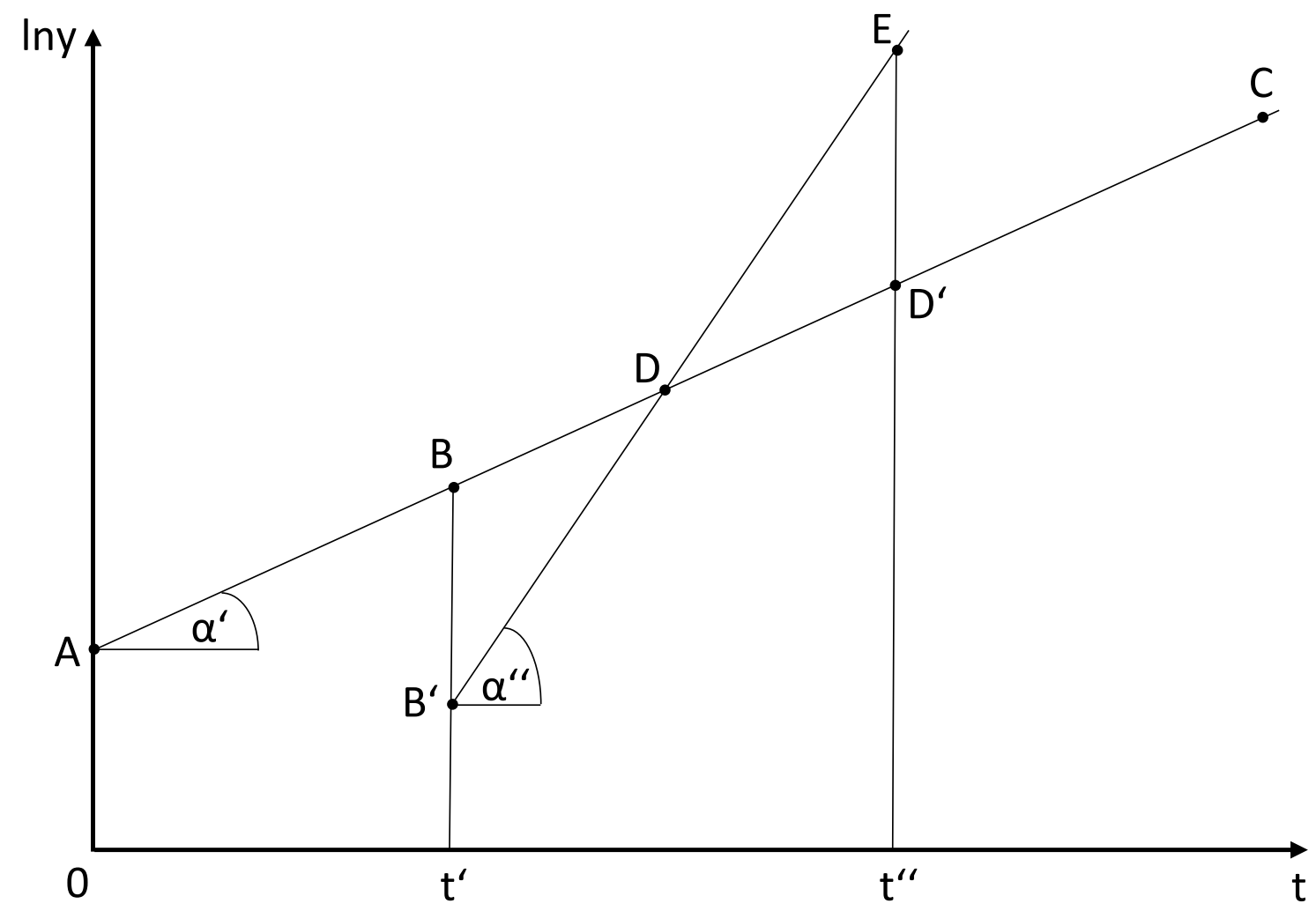

For policy makers, innovation dynamics that are linked to a change of capital income are a serious challenge if there is a rising global innovation race. It is not an attractive idea to reduce the size of the R\&D sector as a means to reduce income inequality and to prevent a rise of the capital income share. Rather, new forms of participation of workers in company equity capital could be considered - here the US, the UK, Sweden and the Netherlands have been active for many years. 


\section{Policy Conclusions}

The analysis presented here has developed a simple model in which the size of the R\&D sector affects the output elasticity of capital positively; and therefore, in the context of a Cobb-Douglas production function, implies that the share of capital income in real gross domestic product will also increase. In the parsimonious model setup, the basic ingredients are a macroeconomic production function in which only a share of $1-\beta^{\prime}$ of workers are producing final output while $\beta^{\prime}$ is the share of workers active in the $R \& D$ sector. $R \& D$ activities can raise the growth rate of knowledge according to a simple progress function in which a productivity parameter $\lambda$ ", as well as an innovation efficiency parameter $\mathrm{v}$, determine the speed of knowledge accumulation (except for the depreciation rate $\lambda^{\prime}$ ). The implication is that a modified neoclassical growth model can explain not only a higher income inequality in a more innovative society - namely the rise of the income share of capital in GDP - but that in the context of a golden rule analysis one can also derive the optimum size of the R\&D sector.

The analysis suggests that policy makers should carefully consider the golden rule implications for two main reasons:

- If the capital intensity k' is lower than k'gold, society will have produced more machinery and equipment than is optimal and this means that there are welfare losses in the form of non-optimum per capita consumption or foregone leisure.

- Moreover, an excessive capital intensity implies additional welfare losses through higher emissions from the production of machinery and equipment (and from running the excess machinery in a more comprehensive modeling approach that takes into account energy as an input in the production function).

While modern endogenous growth theory has generated many new ideas, the model suggests strange results in a setting with a negative real interest rate, since the basic model implies negative growth rates of output and consumption, respectively. For specific parameter settings, the neoclassical growth model is equivalent to the modern growth theory. The advantage of a suitably modified neoclassical growth model is that it allows to easily accommodate a broad range of issues and problems in a simple way, in order to show critical implications in a straightforward way.

There are crucial implications of the model presented if one can decompose the efficiency parameter of the R\&D sector, namely $\lambda$ ", into a domestic component $(\lambda)$ and an element that is related to cumulated foreign direct investment inflows and the share of such inflows in the total capital stock, respectively. Moreover, one may also assume that the intensity of imported intermediate products plays a role (e.g. specifying that $\lambda^{\prime \prime}=\lambda+j^{\prime \prime} j^{\prime}+\alpha^{\prime} \alpha^{*}$ where $j^{\prime}$ is the ratio of imported intermediate inputs to real GDP and $j "$ is a positive parameter). With respect to the envisaged Transatlantic Trade and Investment Partnership (TTIP) between the USA and the European Union, one should clearly look not only into the trade dynamics in the context of broad trade liberalization but also into the implications for foreign direct investment dynamics and innovation (WELFENS/IRAWAN, 2014a; 2014b, have shown that there is a positive link between US foreign direct inflows into the EU and the innovation performance index as measured by the European Commission). Moreover, the progress function may have an international spillover element so that $\mathrm{a}^{*}$ will contribute to raising the progress rate a - not much is known about such transatlantic knowledge spillover and it is also rather opaque how a more consistent 
transatlantic regulatory environment, difficult to achieve given the independence of US political regulators in many fields, could affect international investment and innovation dynamics.

There is a need for empirical research and also for refinements of the neoclassical growth model suggested. Basically, real money balances can be considered in the production function (see WELFENS, 2011) and the role of cumulated foreign direct investment - concerning both inward flows and outward flows - could be included into a more complex and more realistic approach. The new ideas presented here lend themselves to rather easy testing, particularly if one wants to look at the link between the growth rate of total factor producitivity growth and the various critical variables emphasized here; thus the Kaldorian progress function might face a crucial revival.

One may point out here that the golden rule issue, in the context of choosing the optimum size of the R\&D sector, raises some further issues that are not covered here but require future new research. One interesting question is the role of the tax rate and the government budget, respectively. In a simple setup without, debt the government budget constraint requires that government real expenditures $G$ is equal to the tax revenue $\tau Y$. A realistic R\&D sector may be analyzed with a more complex framework that should include the role of government R\&D promotion expenditures ( $G^{\prime}$ as opposed to government consumption $G^{\prime \prime} ; G:=G^{\prime}+G^{\prime \prime}$ ).

Hence, in a broader analytical framework, government has a role in knowledge generation, at the same time one should consider a complex tax optimization issue. If production of output is associated with emissions that contribute to global warming, one may argue that it would be adequate to adopt an income tax rate that is sufficient to cover the administration cost of government and internalizes the negative external effects from production. However, if R\&D activities have positive external effects it would also be adequate that government subsidizes to a certain extent R\&D activities; the relevant income tax rate has to be determined within a modified budget constraint, namely (with $\gamma^{\prime}:=G^{\prime} / Y$ and $\gamma^{\prime \prime}:=G^{\prime \prime} / Y$ ) that $V^{\prime \prime} \beta^{\prime} Y+\gamma^{\prime \prime} Y=\tau f Y$; here $V^{\prime \prime}$ is a government $R \& D$ efficiency parameter in $R \& D$ promotion and $f$ stands for an emission intensity parameter. Obviously, $\gamma^{\prime}=V^{\prime \prime} \beta^{\prime}$ and hence we have $\tau f=V^{\prime \prime} \beta^{\prime}+\gamma^{\prime \prime}$, so that the endogenous nature of the income tax rate would have to be considered as an additional analytical challenge. The savings function would have to be modified adequately and the analysis becomes more complex. However, there are clear perspectives to gain further insights and also to face the problem that the golden rule $\Omega^{\prime}$ is not necessarily compatible with the optimum income tax rate in the sense that the income tax rate internalizes negative external effects of production while allowing to also finance the necessary public administration cost (a VAT tax rate thus might have to be considered additionally). Hence a rich menu of research extensions in a Schumpeterian spirit can be suggested.

At the bottom line, the approach presented suggests a new and interesting way to make the Cobb-Douglas production function richer and more realistic, namely in the context of an R\&D sector that draws on labor as input for new knowledge. While the technological progress function is rather simple in its construction, the steady state solution of the progress rate nevertheless has four key parameters so that some key elements of reality are obviously covered. Future research should consider an explicit microeconomic underpinning for the technological progress function and, naturally, empirical analysis will also be crucial to get a clear view how of realistic and relevant the proposed theoretical innovations are. In an open economy, international R\&D spillover effects could be of particular interest and, in combination with foreign direct investment, a rich array of analytical challenges will have to be faced. As 
emphasized in WELFENS (2011) and WELFENS (2013), foreign direct investment can indeed be included in macroeconomic models as well as growth models, so that future additional research steps should be rather straightforward to implement. In a world economy with economic globalization, the open economy perspectives could indeed generate high marginal benefits of analytical progress in growth and innovation analysis.

As regards the Golden Rule requirement that the savings rate should be equal to the output elasticity of capital - or the capital income share (in an economy with competition in goods markets and input markets - one should point out a practical problem in terms of measurement of the savings ratio; looking at the World Bank's broadly defined adjusted savings rate - which includes expenditures on education and substracts depreciation of natural resources - and the standard savings rate, one finds considerable differences (as is shown in the appendix: without taxation it holds that if the capital intensity exceeds kgold the real interest rate is smaller than the growth rate of output). To the extent that one wants to assess fulfillment of the golden rule on the basis of the difference between the growth rate of output and the long term real interest rate one finds that the US, Japan and the UK are largely in line with the Golden Rule (see appendix 2) while France, China, Canada, India and Indonesia seem to violate the Golden Rule, defined here as the difference of real GDP growth and the real interest rate. In a relatively poor country, such as Indonesia and India, such violation of the Golden Rule can bring serious consequences for part of the population. To the extent that the capital stock per capita exceeds that which would be in line with the Golden Rule capital intensity - e.g. in China - the implication is not only underperformance of consumption per capita but excessive $\mathrm{CO} 2$ emissions as well. While the income tax rate could explain part of the difference between the real growth rate of grosss domestic product and the real interest rate, large swings in this difference over time (as e.g. in France) cannot be explained by income tax changes which are usually rather smooth; a positive income tax rate implies for the Golden Rule that the growth rate of output is smaller than the real interest rate. As regards measurement of the share of income capital, one should point out that the figures presented in Tab. 1 might not give a true picture of the inequality situation. If one would include capital gains on stocks owned by the group of capital income recipients as imputed income, the effective capital income share would clearly rise and a rather consistent positive correlation between the effective income share and the R\&D-GDP ratio - as a proxy for the size of the R\&D sector - could be shown. 


\section{References}

AGHION, P.; HOWITT, P. (2009), The Economics of Growth, Cambridge, MA, MIT Press.

GROSSMAN, G.; HELPMAN, E. (1991), Quality Ladders in the Theory of Growth, Review of Economic Studies, Vol. 58, 43-61.

JONES, C.I. (1995), R\&D-Based Models of Economic Growth, Journal of Political Economy, Vol. 103, 759-784.

JUNGMiTTAG, A. (2015), Techno-Globalization as a New Phenomenon: Theory and Empirical Findings for OECD Countries, paper presented at the AIT-EIIW workshop on Structural Change, Techno-Globalization and Lead Markets, Vienna, March 16, 2015.

KALDOR, N. (1957), A Model of Economic Growth, Economic Journal, Vol. 67, 591-624.

LAURENS, P. ET AL. (2015), The Rate and Motives of the Internationalisation of Large Firm R\&D (1994-2005): Towards a Turing Point?, Research Policy, Vol. 44, 765-776.

OECD Factbook 2011-2012 (2011), OECD Factbook 2011-2012: Economic, Environmental and Social Statistics, OECDiLibrary, December 2011.

PHELPS, E.S. (1961), The Golden Rule of Accumulation: A Fable for Growthmen, American Economic Review, Vol. 51, 638-643.

PIKETTY, T. (2014), Capital in the 21 ${ }^{\text {st }}$ Century, Cambridge, Massachusetts: Belknap Press of Harvard University Press.

RIVERA-BATIZ, L., ROMER, P. (1991), Economic Integration and Endogenous Growth, Quarterly Journal of Economics, Vol. 106, 531-555.

ROMER, P. (1987), Growth Based on Increasing Returns Due to Specialization, American Economic Review, Vol. 77, 56-62.

ROMER, P. (1990), Endogenous Technological Change, Journal of Political Economy, Vol. 98, 71102.

SOLOW, R. M. (1957), Technical change and the aggregate production function, Review of Economics and Statistics (The MIT Press) 39 (3): 312-320.

VON WEIZSÄCKER, C. von (1962), Wachstum, Zins und optimale Investitionsquote, Tübingen: Mohr.

WELFENS, P.J.J. (2011), Innovations in Macroeconomics, 3rd revised and enlarged edition, Heidelberg and New York: Springer.

WELFENS, P.J.J. (2013), Social Security and Economic Globalization, Heidelberg: Springer.

WELFENS, P.J.J. (2014), Issues of modern macroeconomics: new post-crisis perspectives on the world economy, International Economics and Economic Policy, Vol. 11(4), 481-527.

WELFENS, P.J.J.; IRAWAN, T. (2014a), Transatlantic Trade and Investment Partnership: Sectoral and Macroeconomic Perspectives for Germany, the EU and the US, International Economics and Economic Policy, Vol. 11(3), 293-328.

WELFENS, P.J.J.; IRAWAN, T. (2014b), European Product Innovation Dynamics and US Economic Impact: Theory and Empirical Analysis, EIIW working paper No. 207, EIIW at the University of Wuppertal. 


\section{Appendix 1: Deriving the Optimal $B^{\prime}$ for the Golden Age}

(10) $\quad-\beta^{\prime}\left(1-\beta-\beta^{\prime \prime} \beta^{\prime}\right)-\ln \left(\beta+\beta^{\prime \prime} \beta^{\prime}\right)=\ln s$

(10.1) $\beta^{\prime \prime}{\beta^{\prime}}^{2}-(1-\beta) \beta^{\prime}+\ln \left(\beta\left(1+\frac{\beta^{\prime \prime} \beta^{\prime}}{\beta}\right)\right)=\ln (s)$

(10.2) $\beta^{\prime \prime}{\beta^{\prime}}^{2}-(1-\beta) \beta^{\prime}+\frac{\beta^{\prime \prime} \beta^{\prime}}{\beta}=\ln (s)+\ln (\beta)$

(10.3) $\beta^{\prime 2}-\frac{\left((1-\beta) \beta+\beta^{\prime \prime}\right)}{\beta \beta^{\prime \prime}} \beta^{\prime}=\left(\frac{\ln (s)+\ln (\beta)}{\beta \prime \prime}\right)$

(10.4) $\beta^{\prime 2}-\frac{\left((1-\beta) \beta+\beta^{\prime \prime}\right)}{\beta \beta^{\prime \prime}} \beta^{\prime}+\left(\frac{\left((1-\beta) \beta+\beta^{\prime \prime}\right)}{2 \beta \beta \prime \prime}\right)^{2}=\left(\frac{\left((1-\beta) \beta+\beta^{\prime \prime}\right)}{2 \beta \beta^{\prime \prime}}\right)^{2}+\left(\frac{\ln (s)+\ln (\beta)}{\beta \prime \prime}\right)$

(10.5) $\left(\beta^{\prime}-\frac{((1-\beta) \beta+\beta \prime \prime)}{2 \beta \beta \prime \prime}\right)^{2}=\left(\frac{((1-\beta) \beta+\beta \prime \prime}{2 \beta \beta \prime \prime}\right)^{2}+\left(\frac{\ln (s)+\ln (\beta)}{\beta \prime \prime}\right)$

(11) $\beta^{\prime}=\frac{1}{2 \beta \beta^{\prime \prime}}\left[\left((1-\beta) \beta+\beta^{\prime \prime}\right)+\sqrt{\left((1-\beta) \beta+\beta^{\prime \prime}\right)^{2}+4 \beta^{\prime \prime} \beta^{2}(\ln (s)+\ln (\beta))}\right]$ 


\section{Appendix 2: Perspectives on Meeting the Golden Rule in}

Selected Countries (difference refers to real GDP growth minus real interest

rate)

"Real interest rate is the lending interest rate adjusted for inflation as measured by the GDP deflator. The terms and conditions attached to lending rates differ by country, however, limiting their comparability."

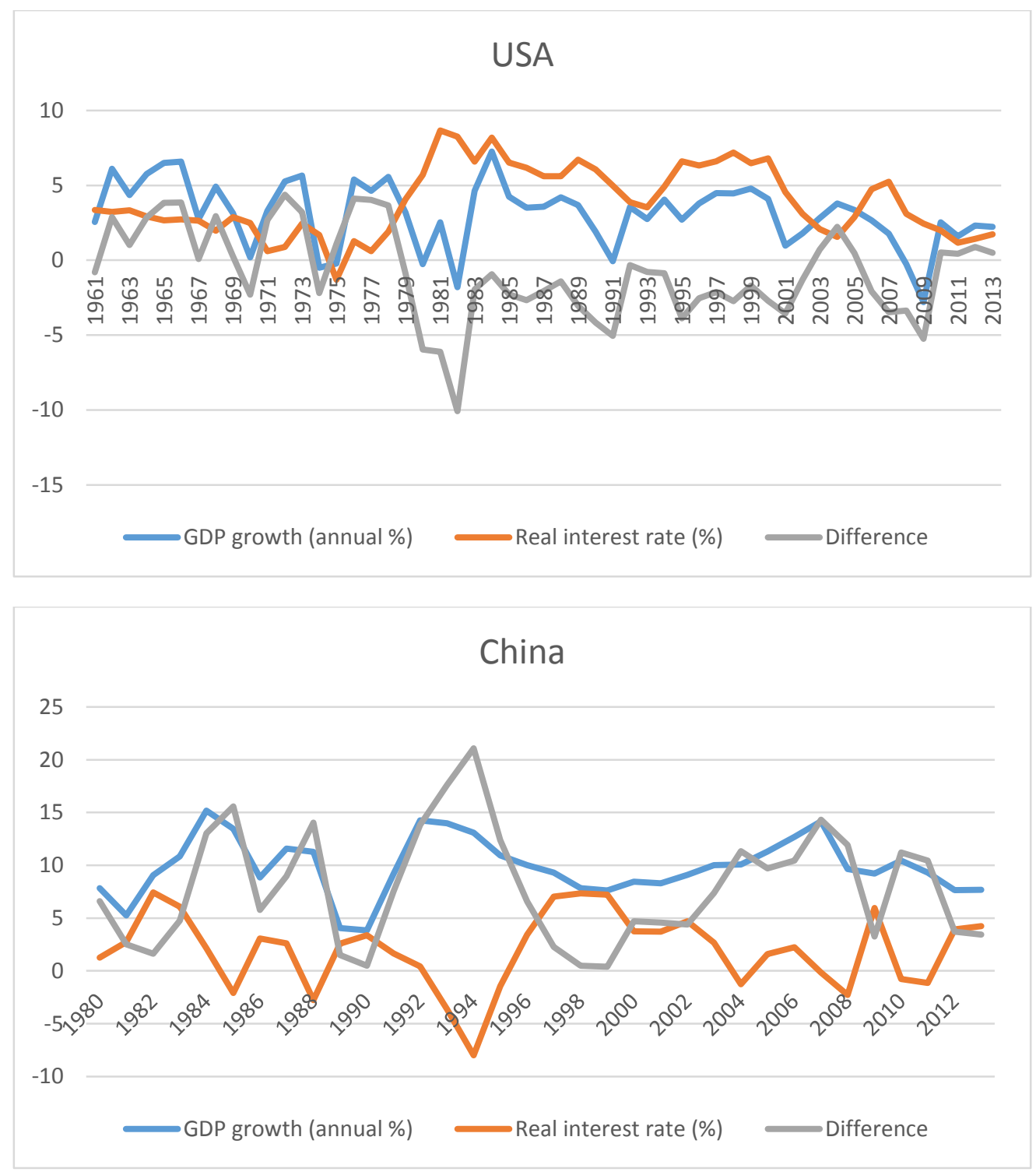




\section{Japan}

15

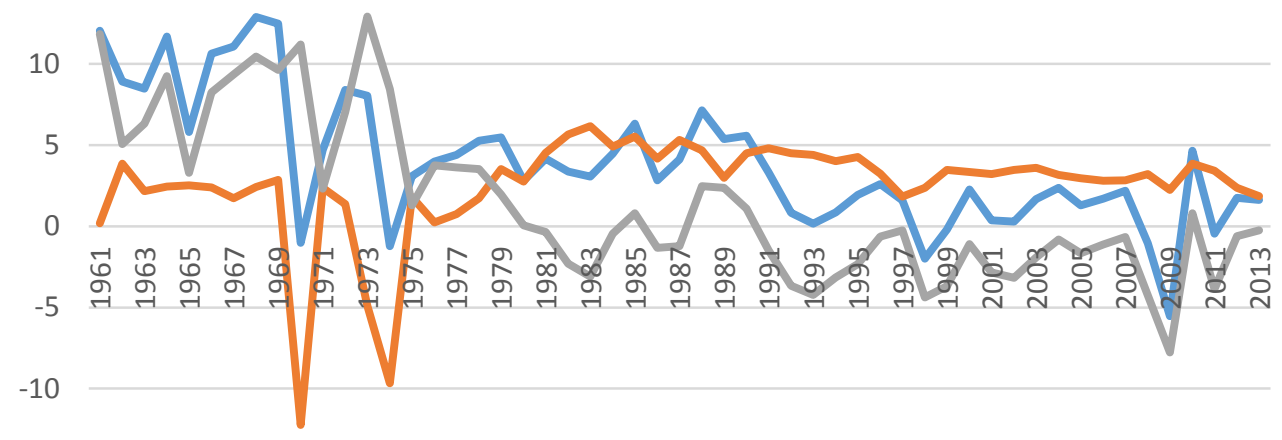

$-15$

$\longrightarrow$ GDP growth (annual \%) —

\section{Germany}

15

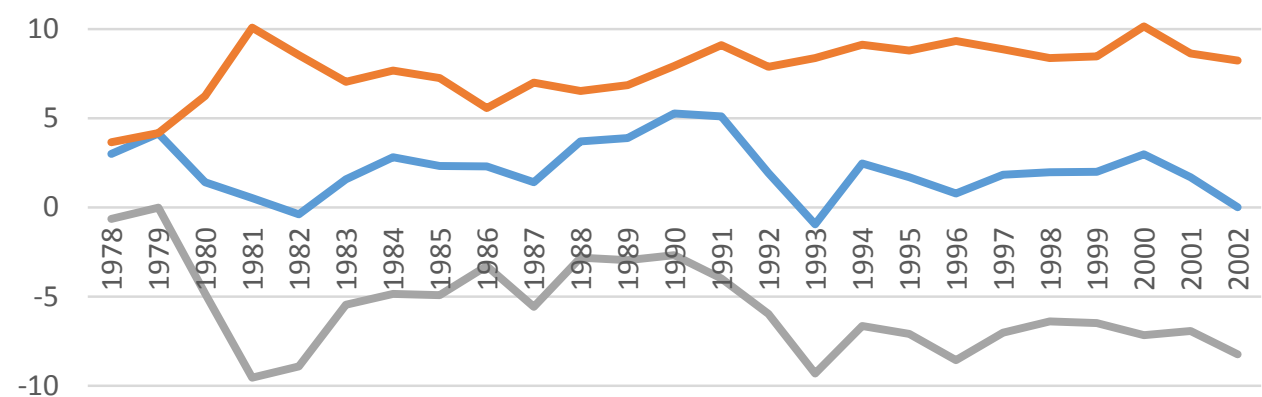

$-15$
GDP growth (annual \%)
Real interest rate (\%)
Difference

\section{France}

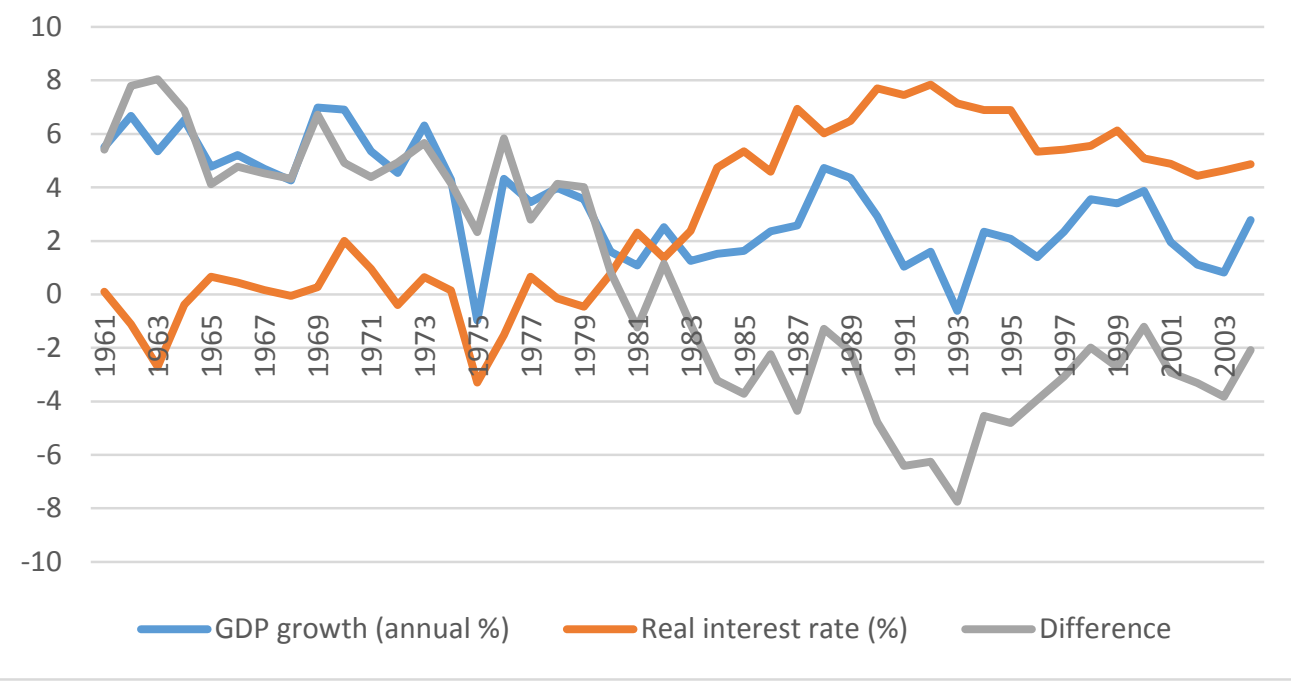



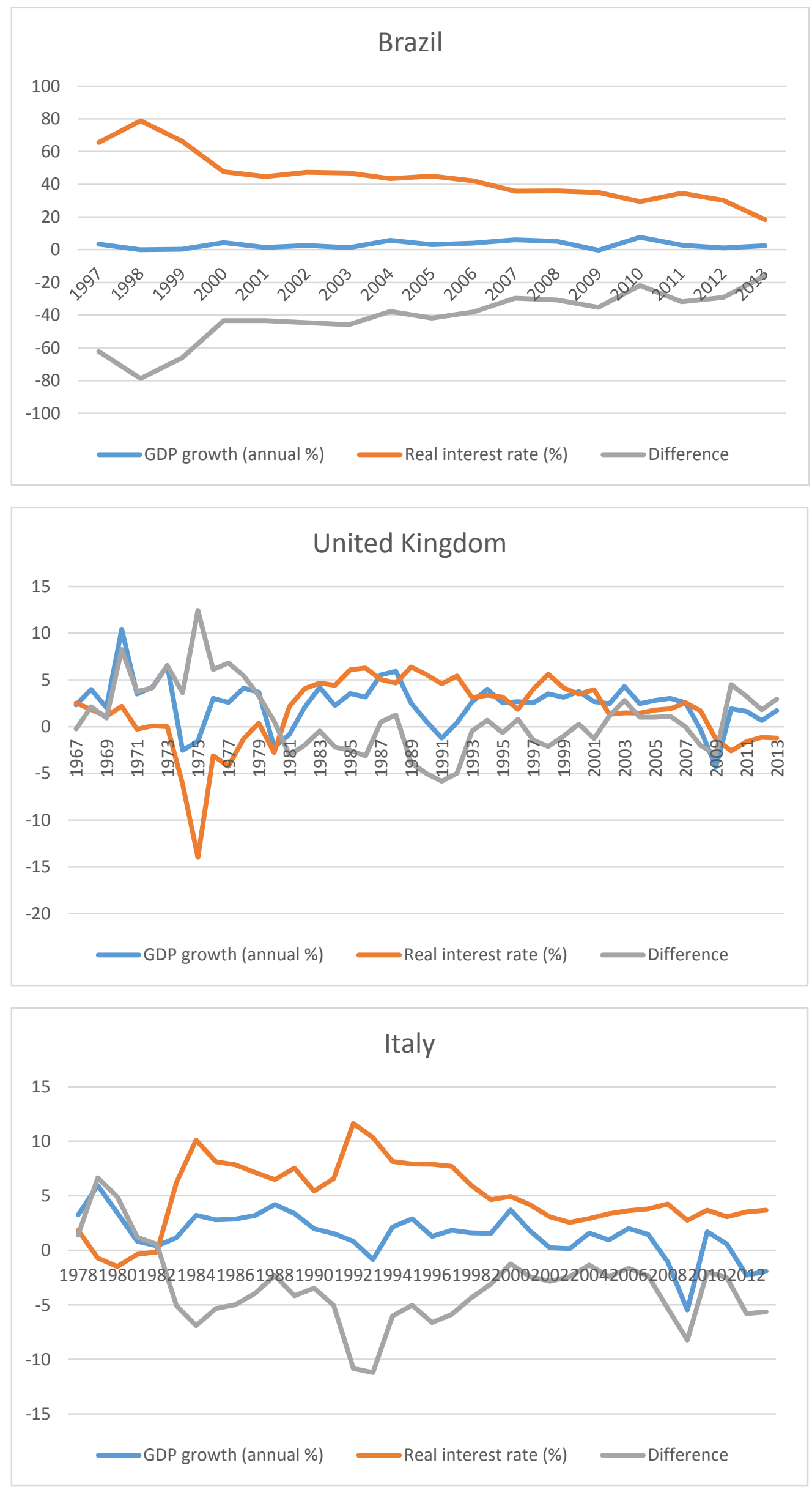


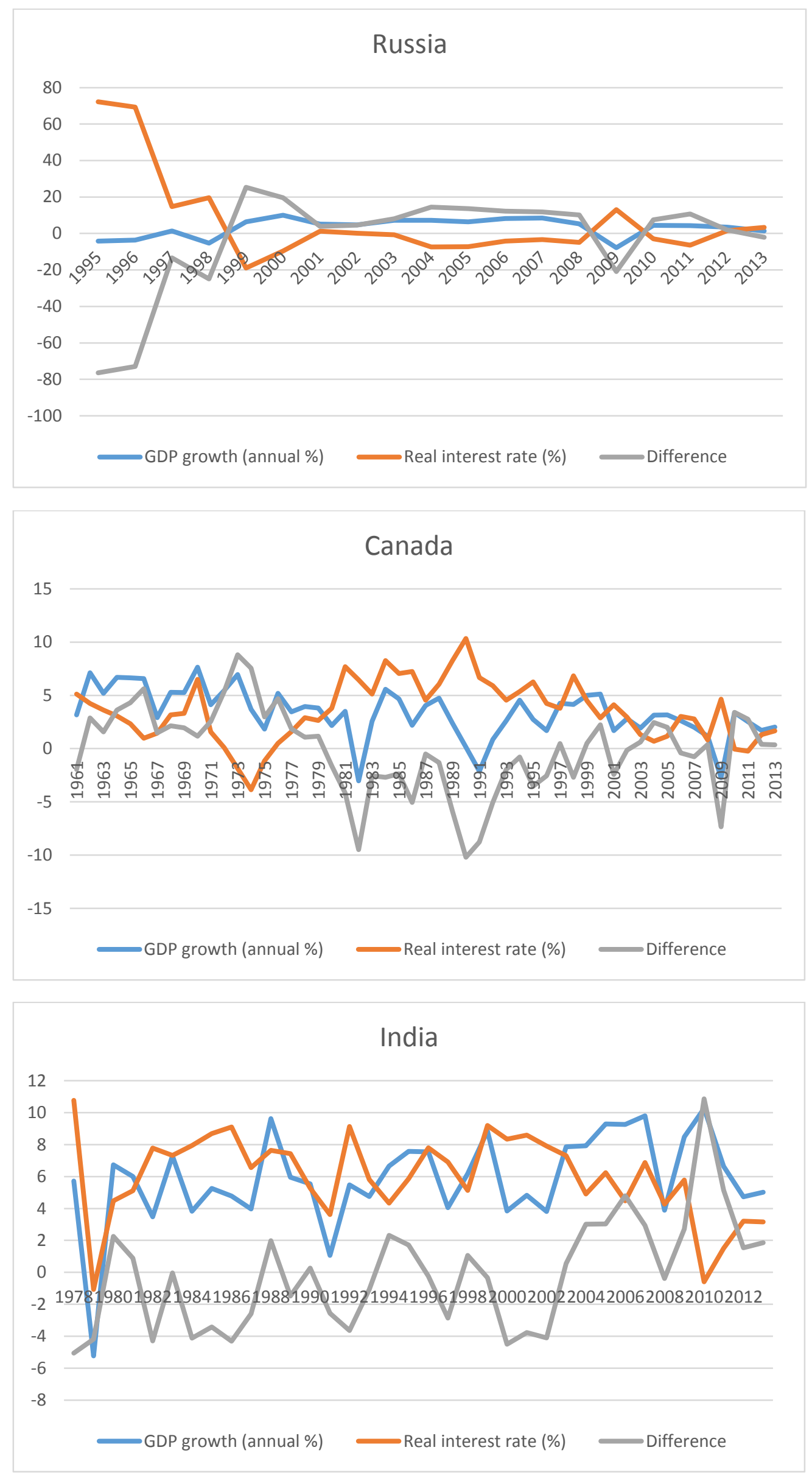




\section{Indonesia}

30

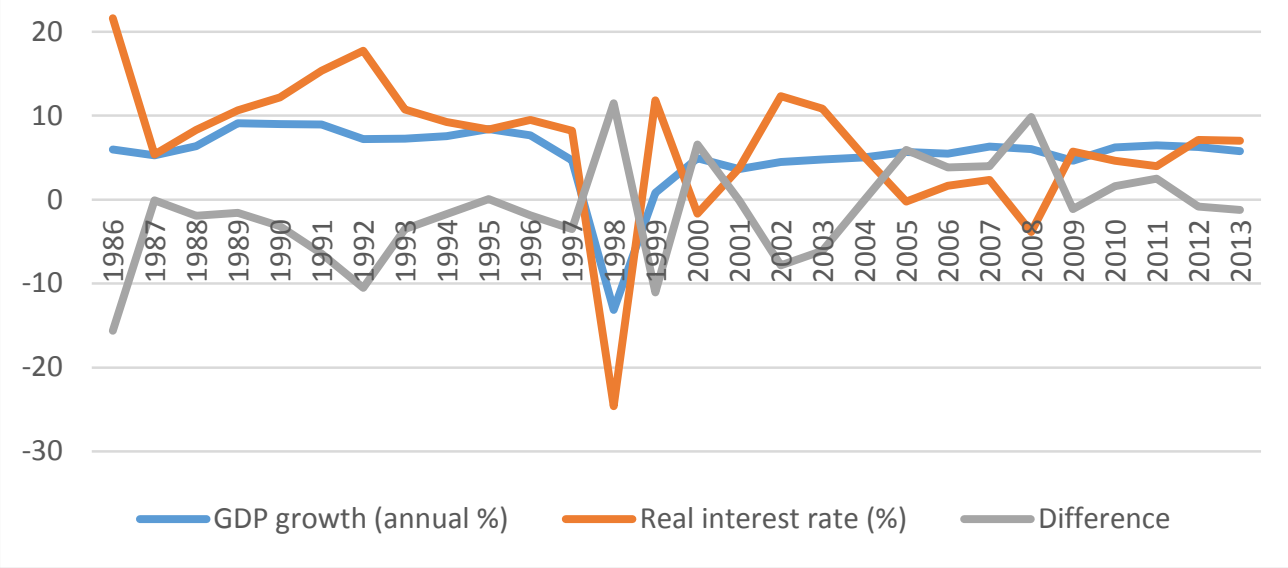

Source: Worldbank 


\section{Appendix 3: Genuine and Gross Savings}

\begin{tabular}{|c|c|c|c|c|c|c|c|c|c|}
\hline \multirow[b]{2}{*}{ Country Name } & \multicolumn{3}{|c|}{$\begin{array}{l}\text { (1) Adjusted net savings, } \\
\text { including particulate } \\
\text { emission damage (\% of } \\
\text { GNI) }\end{array}$} & \multicolumn{3}{|c|}{$\begin{array}{l}\text { (2) Adjusted savings: gross } \\
\text { savings (\% of GNI) }\end{array}$} & \multicolumn{3}{|c|}{$\begin{array}{c}\text { (3) Difference between (1) } \\
\text { and (2) }\end{array}$} \\
\hline & 1990 & 2000 & 2005 & 1990 & 2000 & 2005 & 1990 & 2000 & 2005 \\
\hline Australia & 8.53 & 7.41 & 6.07 & 24.59 & 22.05 & 21.79 & -16.05 & -14.64 & -15.71 \\
\hline Austria & 14.12 & 13.70 & 14.14 & 24.93 & 24.09 & 25.37 & -10.81 & -10.40 & -11.23 \\
\hline Belgium &.. & .. & 13.90 &.. &.. & 24.67 & .. & .. & -10.78 \\
\hline Canada & 9.19 & 13.08 & 11.70 & 18.54 & 24.51 & 25.07 & -9.35 & -11.43 & -13.37 \\
\hline Chile & 0.98 & 2.15 & 2.06 & 25.07 & 21.30 & 25.24 & -24.09 & -19.15 & -23.18 \\
\hline Czech Republic & .. & 7.21 & 9.24 &.. & 27.17 & 26.50 & .. & -19.97 & -17.26 \\
\hline Denmark & 10.77 & 12.50 & 13.83 & 22.23 & 23.72 & 25.15 & -11.46 & -11.23 & -11.31 \\
\hline Estonia & .. & 11.98 & 15.13 & .. & 23.80 & 24.87 & .. & -11.82 & -9.74 \\
\hline Finland & 10.19 & 18.37 & 15.42 & 24.08 & 28.87 & 25.48 & -13.89 & -10.50 & -10.06 \\
\hline France & 11.52 & 13.92 & 11.64 & 19.98 & 20.97 & 19.33 & -8.46 & -7.05 & -7.69 \\
\hline Germany & .. & 8.94 & 10.98 & 22.72 & 20.39 & 22.06 & .. & -11.45 & -11.08 \\
\hline Greece & 7.38 & 4.08 & 1.97 & 18.85 & 14.43 & 12.46 & -11.47 & -10.35 & -10.50 \\
\hline Hungary & 16.97 & 2.91 & 5.45 & 27.18 & 20.48 & 17.65 & -10.21 & -17.56 & -12.20 \\
\hline Iceland & 2.60 & 6.69 & 7.39 & 17.48 & 13.45 & 12.61 & -14.88 & -6.76 & -5.22 \\
\hline Ireland & 16.98 & 20.51 & 22.20 & 24.01 & 28.40 & 29.42 & -7.03 & -7.89 & -7.22 \\
\hline Israel & 11.36 & 11.05 & 13.20 & 22.21 & 18.37 & 22.03 & -10.85 & -7.32 & -8.83 \\
\hline Italy & 8.45 & 9.16 & 7.80 & 21.18 & 20.79 & 20.17 & -12.73 & -11.63 & -12.37 \\
\hline Japan & 20.72 & 10.32 & 8.53 & 33.67 & 27.27 & 25.45 & -12.94 & -16.95 & -16.92 \\
\hline Korea, Rep. & 25.05 & 22.46 & 22.90 & 34.04 & 34.64 & 33.80 & -8.99 & -12.18 & -10.91 \\
\hline Luxembourg & .. & 21.21 & 26.10 & .. & 33.30 & 35.92 & .. & -12.09 & -9.82 \\
\hline Mexico & 7.50 & 12.87 & 10.34 & 20.95 & 21.22 & 21.97 & -13.45 & -8.35 & -11.63 \\
\hline Netherlands & 13.53 & 16.07 & 14.53 & 25.71 & 27.53 & 26.05 & -12.19 & -11.46 & -11.52 \\
\hline New Zealand & 7.97 & 10.82 & 9.29 & 17.33 & 19.95 & 17.89 & -9.36 & -9.13 & -8.60 \\
\hline Norway & 4.72 & 12.58 & 15.76 & 25.68 & 35.87 & 37.53 & -20.96 & -23.29 & -21.78 \\
\hline Poland & .. & 7.74 & 6.99 & .. & 18.89 & 17.31 & .. & -11.15 & -10.32 \\
\hline Portugal & 13.72 & 6.32 & 1.36 & 27.03 & 18.60 & 14.33 & -13.31 & -12.28 & -12.97 \\
\hline $\begin{array}{l}\text { Slovak } \\
\text { Republic }\end{array}$ & .. & 3.88 & 4.31 & .. & 23.40 & 22.03 & .. & -19.52 & -17.73 \\
\hline Slovenia & .. & 11.46 & 14.58 & .. & 24.58 & 25.78 &.. & -13.12 & -11.21 \\
\hline Spain & 11.96 & 12.88 & 11.59 & 22.74 & 22.77 & 22.78 & -10.78 & -9.88 & -11.19 \\
\hline Sweden & 14.39 & 17.62 & 18.83 & 22.72 & 23.17 & 24.85 & -8.33 & -5.54 & -6.02 \\
\hline Switzerland & 16.01 & 18.15 & 19.30 & 31.84 & 31.49 & 31.95 & -15.83 & -13.34 & -12.65 \\
\hline Turkey & 14.44 & 11.55 & 9.16 & 21.93 & 18.20 & 15.78 & -7.50 & -6.65 & -6.62 \\
\hline $\begin{array}{l}\text { United } \\
\text { Kingdom }\end{array}$ & 4.54 & 5.97 & 6.92 & 15.96 & 14.73 & 14.94 & -11.42 & -8.75 & -8.02 \\
\hline United States & 10.01 & 12.31 & 9.15 & 18.95 & 20.31 & 17.75 & -8.94 & -8.00 & -8.60 \\
\hline
\end{tabular}

Source: Worldbank, World Development Indicators 


\section{Appendix 4: Info on Tax Revenue}

Table 8: $\quad$ Total Tax Revenue as \% of GDP

\begin{tabular}{|c|c|c|c|c|}
\hline Country & 1995 & 2000 & 2005 & Change $(\Delta)$ \\
\hline Australia & 28.2 & 30.4 & 29.9 & 1.7 \\
\hline Austria & 41 & 42.1 & 40.8 & -0.2 \\
\hline Belgium & 42.8 & 43.8 & 43.4 & 0.6 \\
\hline Canada & 34.9 & 34.9 & 32.3 & -2.6 \\
\hline Chile & 18.4 & 18.8 & 20.7 & 2.3 \\
\hline Czech Republic & 34.9 & 32.5 & 34.5 & -0.4 \\
\hline Denmark & 48 & 48.1 & 49.5 & 1.5 \\
\hline Estonia & 36.2 & 30.9 & 30.4 & -5.8 \\
\hline Finland & 44.5 & 45.8 & 42.1 & -2.4 \\
\hline France & 41.9 & 43.1 & 42.8 & 0.9 \\
\hline Germany & 36.2 & 36.3 & 33.9 & -2.3 \\
\hline Greece & 27.6 & 33.1 & 31.3 & 3.7 \\
\hline Hungary & 41 & 38.7 & 36.8 & -4.2 \\
\hline Iceland & 30.4 & 36.2 & 39.4 & 9 \\
\hline Ireland & 31.8 & 30.9 & 29.5 & -2.3 \\
\hline Israel & 35.2 & 35.6 & 34.3 & -0.9 \\
\hline Italy & 38.6 & 40.6 & 39.1 & 0.5 \\
\hline Japan & 26.4 & 26.6 & 27.3 & 0.9 \\
\hline Korea & 19 & 21.5 & 22.5 & 3.5 \\
\hline Luxembourg & 35.3 & 37.2 & 38.2 & 2.9 \\
\hline Mexico & 14.9 & 16.5 & 17.7 & 2.8 \\
\hline Netherlands & 39 & 36.8 & 36.4 & -2.6 \\
\hline New Zealand & 35.8 & 32.9 & 36.4 & 0.6 \\
\hline Norway & 40.9 & 42.6 & 43.2 & 2.3 \\
\hline Poland & 36.1 & 32.7 & 32.9 & -3.2 \\
\hline Portugal & 28.9 & 30.6 & 30.2 & 1.3 \\
\hline Slovak Republic & 39.6 & 33.6 & 30.8 & -8.8 \\
\hline Slovenia & 38.4 & 36.6 & 38 & -0.4 \\
\hline Spain & 31.3 & 33.4 & 35.2 & 3.9 \\
\hline Sweden & 45.6 & 49 & 46.6 & 1 \\
\hline Switzerland & 25.5 & 27.6 & 26.5 & 1 \\
\hline Turkey & 16.8 & 24.2 & 24.3 & 7.5 \\
\hline United Kingdom & 32.1 & 34.7 & 33.8 & 1.7 \\
\hline United States & 26.7 & 28.4 & 26.1 & -0.6 \\
\hline OECD - Average & 33.6 & 34.3 & 34 & 0.4 \\
\hline
\end{tabular}

Source: OECD Database 